\title{
New Insights into the Regulation of Aquaporins by the Arbuscular Mycorrhizal Symbiosis in Maize Plants Under Drought Stress and Possible Implications for Plant Performance
}

\author{
Gloria Bárzana, ${ }^{1}$ Ricardo Aroca, ${ }^{1}$ Gerd Patrick Bienert, ${ }^{2}$ François Chaumont, ${ }^{2}$ and \\ Juan Manuel Ruiz-Lozano' \\ ${ }^{1}$ Departamento de Microbiología del Suelo y Sistemas Simbióticos, Estación Experimental del Zaidín (CSIC), Profesor \\ Albareda number 1, 18008 Granada, Spain; ${ }^{2}$ Institut des Sciences de la Vie, Université Catholique de Louvain, Croix du \\ Sud 4-L7.07.14. B-1348, Louvain-la-Neuve, Belgium
}

Submitted 12 September 2013. Accepted 23 November 2013.

The relationship between modulation by arbuscular mycorrhizae (AM) of aquaporin expression in the host plant and changes in root hydraulic conductance, plant water status, and performance under stressful conditions is not well known. This investigation aimed to elucidate how the AM symbiosis modulates the expression of the whole set of aquaporin genes in maize plants under different growing and drought stress conditions, as well as to characterize some of these aquaporins in order to shed further light on the molecules that may be involved in the mycorrhizal responses to drought. The AM symbiosis regulated a wide number of aquaporins in the host plant, comprising members of the different aquaporin subfamilies. The regulation of these genes depends on the watering conditions and the severity of the drought stress imposed. Some of these aquaporins can transport water and also other molecules which are of physiological importance for plant performance. AM plants grew and developed better than non-AM plants under the different conditions assayed. Thus, for the first time, this study relates the well-known better performance of AM plants under drought stress to not only the water movement in their tissues but also the mobilization of $\mathbf{N}$ compounds, glycerol, signaling molecules, or metalloids with a role in abiotic stress tolerance. Future studies should elucidate the specific function of each aquaporin isoform regulated by the AM symbiosis in order to shed further light on how the symbiosis alters the plant fitness under stressful conditions.

Drought has a major impact on plant growth and development, limiting crop production throughout the world. The severity of drought is unpredictable because it depends on many factors such as occurrence and distribution of rainfall, evapora-

Current address for G. P. Bienert: Leibniz Institute of Plant Genetics and Crop Plant Research, Corrensstrasse 3. 06466 Gatersleben, Germany

Corresponding author: J. M. Ruiz-Lozano; Telephone: +34 958181600; E-mail: juanmanuel.ruiz@eez.csic.es

* The $\boldsymbol{e}$-Xtra logo stands for "electronic extra" and indicates that four supplementary figures and two supplementary tables are published online.

(C) 2014 The American Phytopathological Society tive demands, and moisture storing capacity of soils (Farooq et al. 2009). In addition, global climate change is contributing to spread the problems of water deficit to regions where drought was negligible in the past. Thus, understanding mechanisms that enhance efficient use of water by plants and drought tolerance is fundamental to improve crops and to guarantee world food production (Chaves and Oliveira 2004).

When a soil dries, its water potential decreases, and this hampers the uptake of water by plant roots (Ouziad et al. 2006). Under such conditions, a series of physical and physiological changes aimed to counter the effects of drought are produced for both long-term adaptations, accounted for by morphological changes, as well as short-term effects, a process in which aquaporins participate (Luu and Maurel 2005). Aquaporins are membrane intrinsic proteins that facilitate and regulate the passive movement of water molecules down a water potential gradient (Maurel et al. 2008). In plants, aquaporins comprise a large and diverse protein family composed of 31 to 71 different genes, depending on the plant species, and subdivided into five subgroups based on their amino acid sequence similarity (Maurel et al. 2008; Park et al. 2010). The discovery of aquaporins in plants has significantly changed the understanding of plant water relations and, in recent years, much effort has been concentrated on investigating the function and regulation of aquaporins. High levels of aquaporin expression were shown in tissues with high water fluxes across membranes (e.g., in fast-growing regions in shoots and leaves but also in roots where water uptake occurs). Hence, aquaporins seem to play a specifically important role in controlling transcellular water transport and the hydraulic conductance in plant tissues (Javot and Maurel 2002). Nevertheless, despite intensive research in this field, the relationship that exists between aquaporins and plant responses to water deficit still remains elusive and with contradictory results.

The selectivity filters of plant aquaporins show high divergence (Sui et al. 2001), suggesting a great functional diversity (Bansal and Sankararamakrishnan 2007; Wallace et al. 2002). It has become increasingly clear that some aquaporins do not exhibit a strict specificity for water and can transport other small neutral molecules such as urea (Gerbeau et al. 1999; Liu et al. 2003b), ammonia $\left(\mathrm{NH}_{3}\right)$ (Jahn et al. 2004; Loque et al. 2005), carbon dioxide $\left(\mathrm{CO}_{2}\right)$ (Uehlein et al. 2003), boric acid (Mitani et al. 2008), hydrogen peroxide $\left(\mathrm{H}_{2} \mathrm{O}_{2}\right)$ (Bienert et al. 2007), silicic acid (Ma and Yamaji 2006), and some other 
molecules with physiological significance (Bienert et al. 2008), highlighting the paramount relevance of aquaporins for plant physiology. In particular, the ability of aquaporins to transport molecules such as urea and $\mathrm{NH}_{3}$ has pointed to important roles for aquaporins in nitrogen metabolism. The gas $\mathrm{CO}_{2}$ membrane diffusion through aquaporins suggests their involvement in carbon fixation. The ability of aquaporins to transport $\mathrm{H}_{2} \mathrm{O}_{2}$ points to potential roles in stress signaling and responses. Boron (B) is closely related with nutrition and structural development, and silicon ( $\mathrm{Si}$ ) uptake and metabolism seem to be crucial for responses to biotic and abiotic stresses (Maurel 2007).

In nature, most plants establish a symbiotic interaction with arbuscular mycorrhizal (AM) fungi, and these fungi exist in most agricultural soils worldwide (Varma 2008). By AM symbiosis, plants get from the fungus nutrients and water resources less available to the plant roots, while the fungus receives carbon compounds from the plant and finds an ecological niche to complete its life cycle (Koide and Mosse 2004). At the same time, AM symbiosis enhances plant tolerance to different abiotic stresses with osmotic components such as drought and salinity (Augé 2001; Ruiz-Lozano et al. 2006; Ruiz-Lozano et al. 2012).

The AM symbiosis is a good example for the extensive morphological alterations that plant root cells undergo in order to accommodate the presence of an endophytic symbiont and most of these changes concern vacuolar or cytoplasmic membrane systems. Thus, Krajinski and associates (2000) hypothesized a variation of expression patterns concerning genes that encode membrane-associated proteins such as the aquaporins. In addition, AM symbiosis has the capacity to alter root hydraulic properties (Bárzana et al. 2012; Khalvati et al. 2005). Thus, it is not surprising that AM fungi can also change plant aquaporin gene expression and protein accumulation. Indeed, the importance of aquaporins for both nutrient and water exchanges during mycorrhizal symbiosis has been recently acknowledged (Maurel and Plassard 2011; Ruiz-Lozano and Aroca 2010).

The results obtained by our research group in recent years show that an aquaporin gene can respond differently depending on the specific characteristics of the applied osmotic stress and the presence or absence of the AM fungus in the roots of the host plant (Aroca et al. 2007). However, the number of aquaporin genes studied thus far with respect to AM symbiosis and drought has been very limited and in different host plants (two in soybean, two in lettuce, four in bean, four in tomato, and seven in maize). Thus, there is still an important lack of knowledge about the modulation of expression for most aquaporin genes by the AM symbiosis, as well as about the relationship among this modulation and changes in root hydraulic conductance, plant water status, and plant tolerance to water deficit (Ruiz-Lozano et al. 2006). Indeed, it has been proposed that, to reach correct conclusions regarding the role of aquaporins in particular environmental conditions, the behavior of the whole aquaporin family present in the plant should be analyzed (Alexandersson et al. 2005; Maurel 2007). In addition, plant stress responses vary greatly depending upon the growing conditions and the intensity, rate, and duration of the water deficit exposure. For such reasons, the first objective of this investigation was to elucidate in which way the AM symbiosis modulates the expression of the whole set of aquaporin genes present in a plant under both optimal and drought stress conditions. For that, two independent experiments were carried out, with plants subjected to drought stress treatments of different intensity and duration. The second objective aimed to test the hypothesis of whether the aquaporins regulated by AM symbiosis transport only water or have the capacity to transport other molecules of physiological interest for the plant. Thus, we characterized some of these aquaporins in order to shed further light on the molecules that could be involved in the mycorrhizal responses to drought. To achieve these objectives, maize (Zea mays L.) was selected as a host plant species because i) maize is a basic crop for human supply, whose production is negatively affected by drought (Campos et al. 2004); ii) this plant has been shown to benefits from AM symbiosis under drought stress (Boomsma and Wyn 2008; Ruiz-Lozano et al. 2009); and iii) the whole aquaporin gene family of maize has been already described (Chaumont et al. 2001).

\section{RESULTS}

Biomass production and symbiotic development.

In both short-term and sustained drought stress experiments, AM plants grew more than non-AM plants (Table 1). In experiment 1 , the growth enhancement by AM inoculation, estimated by the shoot dry weight, was 40 and $35 \%$ under wellwatered and drought stress conditions, respectively. In experiment 2, the growth enhancement was 56 and $26 \%$ under wellwatered and drought stress conditions, respectively. Differences in growth between the two experiments were caused by the differences in soil and in the growing conditions used in the two experiments.

Uninoculated control plants did not show mycorrhizal colonization (Table 1). In experiment 1, AM plants exhibited over $69 \%$ of root colonization. In experiment 2 , the rate of AM root colonization was over $76 \%$. In both cases, no effect of water treatments on this parameter was observed.

\section{Sap flow rate and osmotic and hydrostatic root hydraulic conductance.}

In both experiments, drought reduced the sap flow rate (Jv) and the osmotic root hydraulic conductance (Lo) in non-AM plants (Fig. 1A). This diminution was approximately 70 and $50 \%$ (experiments 1 and 2) for $\mathrm{Jv}$ and $75 \%$ for Lo compared with values under well-watered conditions.

Mycorrhization had different effects in both experiments. In experiment 1, no effect on $\mathrm{Jv}$ and Lo under well-watered conditions was observed. Under short-term drought stress conditions, AM plants did not show any decrease in Jv or Lo by drought treatment, having, in both cases, higher values than

Table 1. Shoot fresh and dry weight (SFW and SDW [grams per plant]) and percentage of arbuscular mycorrhizal (AM) root length in two independent experiments with maize plants cultivated under well-watered conditions or subjected to short-term or sustained drought stress ${ }^{\mathrm{z}}$

\begin{tabular}{|c|c|c|c|c|c|c|}
\hline \multirow[b]{2}{*}{ Treatment } & \multicolumn{3}{|c|}{ Short-term drought } & \multicolumn{3}{|c|}{ Sustained drought } \\
\hline & SFW (g) & SDW (g) & $\operatorname{AM}(\%)$ & SFW (g) & SDW (g) & $\mathbf{A M}(\%)$ \\
\hline \multicolumn{7}{|l|}{ Well-watered } \\
\hline Control & $8.75 \mathrm{c}$ & $1.50 \mathrm{~b}$ & $0.0 \mathrm{~b}$ & $28.13 \mathrm{~b}$ & $3.48 \mathrm{~b}$ & $0.0 \mathrm{~b}$ \\
\hline Rhizophagus intraradices & $14.38 \mathrm{a}$ & $2.11 \mathrm{a}$ & $74.3 \mathrm{a}$ & $39.51 \mathrm{a}$ & $5.44 \mathrm{a}$ & $76.1 \mathrm{a}$ \\
\hline \multicolumn{7}{|l|}{ Drought } \\
\hline Control & $8.70 \mathrm{c}$ & $1.45 \mathrm{~b}$ & $0.0 \mathrm{~b}$ & $25.35 \mathrm{~b}$ & $3.78 \mathrm{~b}$ & $0.0 \mathrm{~b}$ \\
\hline R. intraradices & $12.57 \mathrm{~b}$ & $1.95 \mathrm{a}$ & $69.2 \mathrm{a}$ & $28.04 \mathrm{~b}$ & $4.75 \mathrm{a}$ & $78.1 \mathrm{a}$ \\
\hline
\end{tabular}

${ }^{\mathrm{z}}$ Means followed by different letters are significantly different $(P<0.05)$ as determined by Duncan's multiple range and least significant difference tests $(n=6)$. 
non-AM plants (Fig. 1A). In experiment 2, the mycorrhization itself strongly reduced $\mathrm{JV}$ and Lo under well-watered conditions, with no further changes in this parameter when plants were subjected to sustained drought but showing lower values than non-AM plants (Fig. 1B).

In experiment 2, we measured hydrostatic root hydraulic conductance (Lh) in order to analyze the different water transport pathways in AM and non-AM plants.

AM inoculation significantly decreased Lh under wellwatered conditions compared with non-AM plants (Fig. 1B). This decrease was approximately $60 \%$ and matched the diminution of Jv and Lo observed previously. Drought stress produced a significant decrease of $\mathrm{Lh}$ in non-AM plants, with a diminution of approximately $50 \%$. In contrast, in AM plants, drought stress caused an increase of the $\mathrm{Lh}$, reaching values similar to those of well-watered non-AM plants.

\section{Aquaporin gene expression.}

Results showed that the expression of several aquaporin genes was not significantly affected by the AM symbiosis under either well-watered or the two drought stress conditions assayed (data not shown). However, the expression of 16 aquaporin genes was regulated by the AM symbiosis but in different ways depending on the water treatment. Thus, the expression of these aquaporin genes could be grouped into six different patterns, which include representatives of the different plant aquaporin subfamilies (Fig. 2; Supplementary Fig. S1).

Pattern 1. ZmPIP1;1, ZmPIP1;3, and ZmNIP2;2 genes were included into this expression pattern (Fig. 2). In both experiments, mycorrhization caused a downregulation of their expression when plants were cultivated under well-watered conditions. Under short-term drought stress, no significant differences in gene expression could be observed between AM and non-AM plants. In contrast, under sustained drought stress, mycorrhization kept lower the expression of the three genes compared with non-AM plants. It is noteworthy that ZmNIP2;2 expression was downregulated by a short-term drought stress in nonAM plants, whereas it was considerably upregulated in these plants when subjected to sustained drought stress.

Pattern 2. Only ZmTIP1;2 followed this expression pattern (Fig. 2). In experiment 1, the gene was downregulated by both mycorrhization and short-term drought stress. In contrast, in
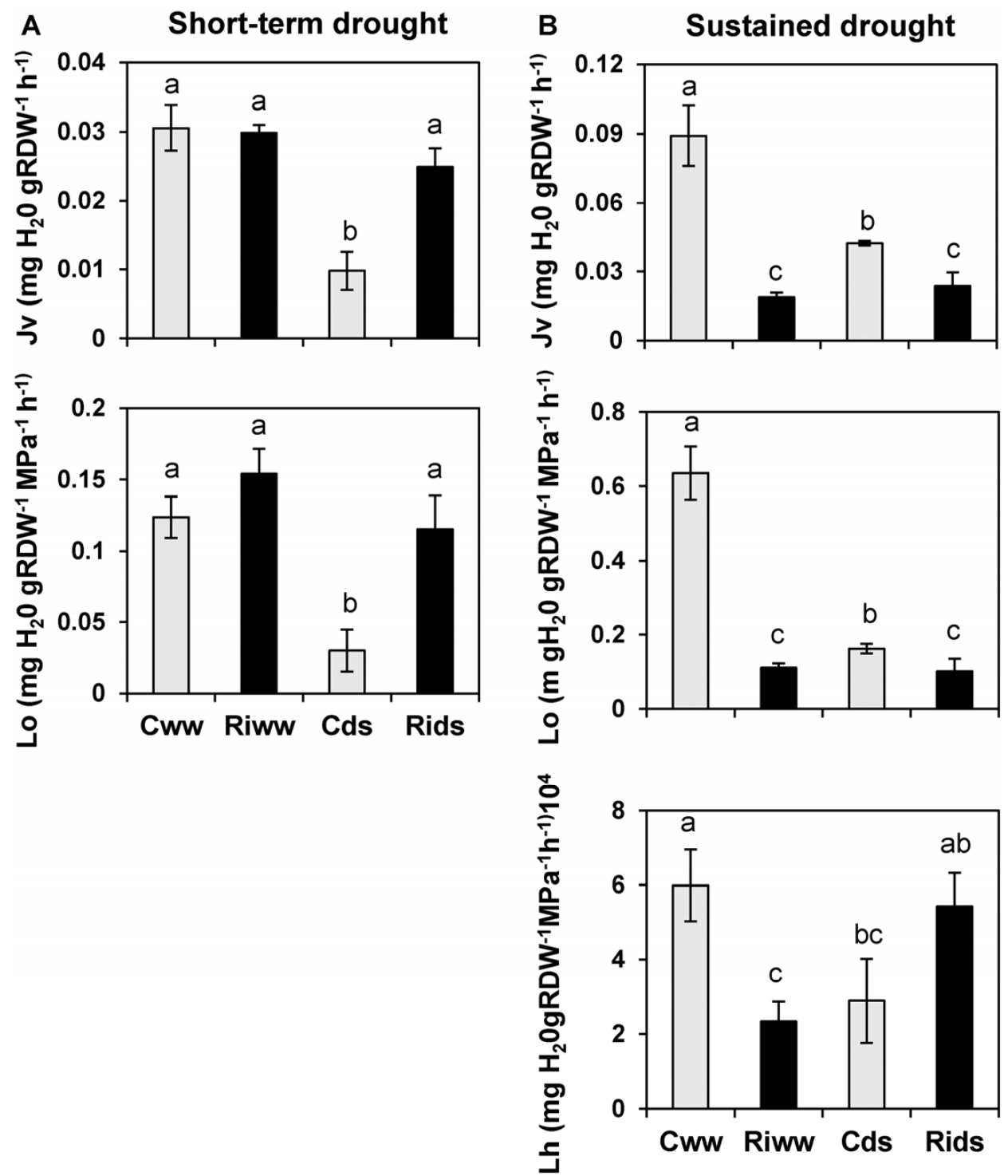

Fig. 1. Free exuded sap flow rate (Jv), osmotic root hydraulic conductance (Lo), and hydrostatic root hydraulic conductance (Lh) of maize plants. A, Experiment 1, with well-watered plants (ww) or plants subjected to short-term drought stress (ds). B, Experiment 2, with ww or ds plants. Plants remained as uninoculated controls (C, open bars) or were inoculated with Rhizophagus intraradices (Ri, black bars). Means followed by different letters are significantly different $(P<0.05)$ as determined by Duncan's multiple range and least significant difference tests $(n=6$ for Jv and Lo, $n=4$ for Lh). 
AM plants, the drought stress did not affect the expression of this gene. In experiment 2, the expression level of ZmTIP1;2 was similar in AM and non-AM plants when plants were cultivated under well-watered conditions. The sustained drought stress did not affect the expression of this gene in non-AM plants but reduced it in AM plants.

Pattern 3. ZmPIP1;4, ZmPIP2;4, ZmNIP2;1, and ZmTIP2;3 were grouped into this expression pattern (Fig. 2). In both experiments, mycorrhization caused a downregulation of their expression when plants were cultivated under well-watered conditions. When plants were subjected to short-term drought stress conditions, the expression of three of these genes was downregulated in non-AM plants. In contrast, mycorrhization upregulated the expression of the four genes under these conditions. In experiment 2 , in addition to the downregulation by mycorrhization already observed under well-watered conditions, the drought stress also caused a downregulation of the expression of the four aquaporin genes in non-AM plants. Under sustained drought stress conditions, the expression of these genes was similar in AM and non-AM plants.

Pattern 4. ZmPIP2;2 and ZmTIP1;1 genes were grouped into this expression pattern (Fig. 2). In both experiments, mycorrhization downregulated the expression of both genes when plants were cultivated under well-watered conditions. Mycorrhization caused an upregulation of these genes when plants

\section{PATTERN 1}
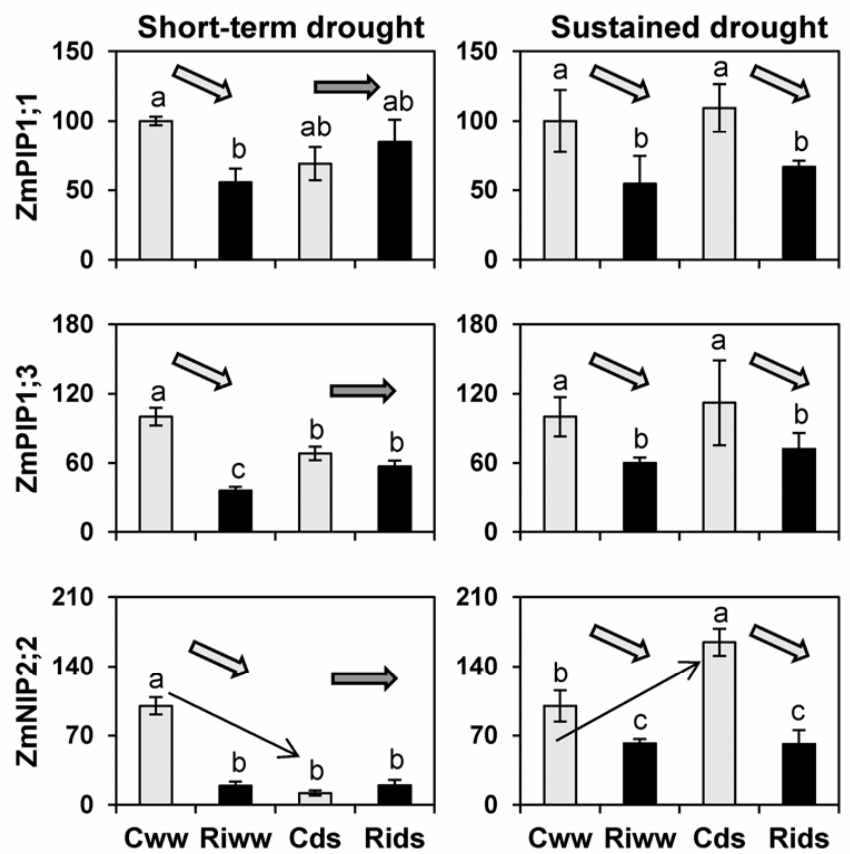

\section{PATTERN 2}

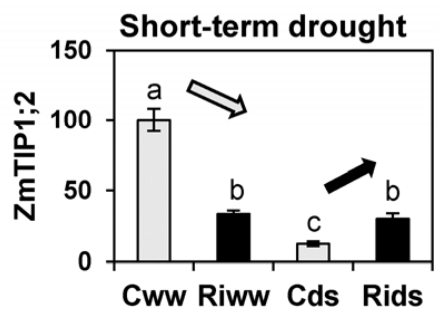

were subjected to short-term drought stress. The contrary occurred when plants were subjected to sustained drought stress. Non-AM plants enhanced the expression of ZmTIP1;1 when subjected to sustained drought stress.

Pattern 5. ZmPIP1;2, ZmPIP1;6, and ZmSIP2;1 were grouped into this expression pattern (Fig. 2). In both experiments, mycorrhization did not significantly affect their expression levels when plants were cultivated under well-watered conditions. When plants were subjected to short-term drought stress, the expression of ZmPIPI;2 and ZmSIP2;1 was downregulated in non-AM plants, whereas that of ZmPIP1;6 was upregulated in these plants. In any case, AM roots had higher expression of these three genes than non-AM roots. In contrast, when plants were subjected to a sustained drought stress, mycorrhization did not significantly alter the expression levels of these aquaporins.

Pattern 6. ZmNIP1;1, ZmTIP4;1, and ZmTIP4;2 genes were included in this expression group (Fig. 2). In experiment 1, mycorrhization caused a downregulation of these aquaporin genes under well-watered conditions. No significant changes in expression due to mycorrhization were observed when plants were subjected to short-term drought stress. In experiment 2 , the opposite was observed, because no significant changes in gene expression due to mycorrhization were found under wellwatered conditions whereas an upregulation of the three genes

\section{PATTERN 3}
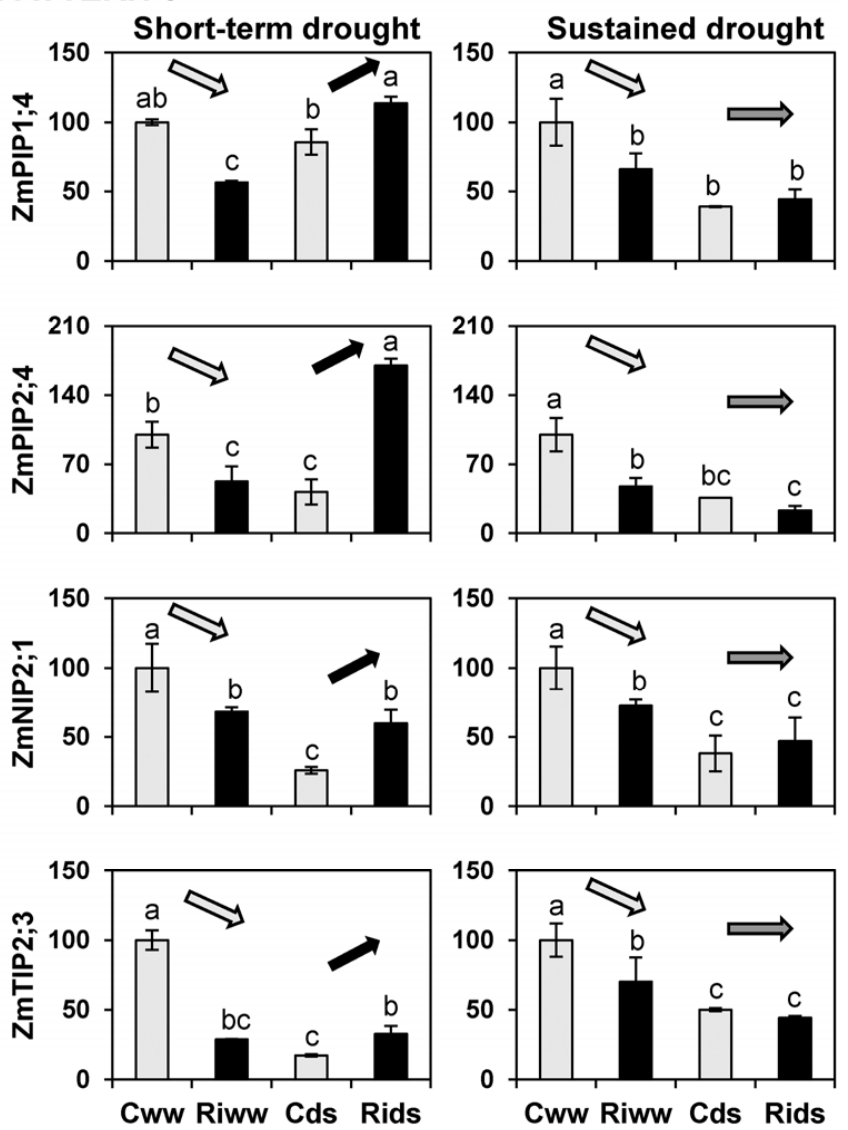

Continued on following page

Fig. 2. Root expression (in relative units) patterns for maize aquaporin genes showing regulation by the arbuscular mycorrhizal (AM) symbiosis and shortterm or sustained drought stress treatments. $\mathrm{C}=$ uninoculated control, $\mathrm{Ri}=$ inoculated with Rhizophagus intraradices, ww $=$ well-watered plants, and ds $=$ plants subjected to short-term drought stress The mean and standard error for three independent reverse-transcriptase polymerase chain reaction experiments, each in triplicate, are shown. Means followed by different letters are significantly different $(P<0.05)$ as determined by Duncan's multiple range and least significant difference tests. 
by mycorrhization was found when plants were subjected to sustained drought stress. It is noteworthy that the three aquaporin genes were downregulated in non-AM plants by the shortterm drought stress but unaffected in these plants by the sustained drought stress. An exception was ZmNIP1;1, which was more expressed in non-AM plants by sustained drought stress.

\section{Protein accumulation.}

Within each expression pattern group, the accumulation of one or more proteins was analyzed, except for patterns 1 and 6 , for which we could not get an adequate antibody. The selection was based on their high expression levels or the particular interest of their expression pattern. Thus, ZmPIP1;2, ZmPIP2;1-PIP2;2, ZmPIP2;3-PIP2;4, ZmPIP2;5, ZmTIP1;1, and ZmTIP1;2 were analyzed.

In experiment 1 , mycorrhization itself decreased the accumulation of the analyzed aquaporins, such decrease being more significant under stress conditions (Fig. 3). In experiment 2 , the same effect by mycorrhization was observed under wellwatered conditions (Fig. 3). In non-AM plants, sustained drought stress caused a diminution of ZmPIP1;2, ZmPIP2;3-

\section{PATTERN 4}
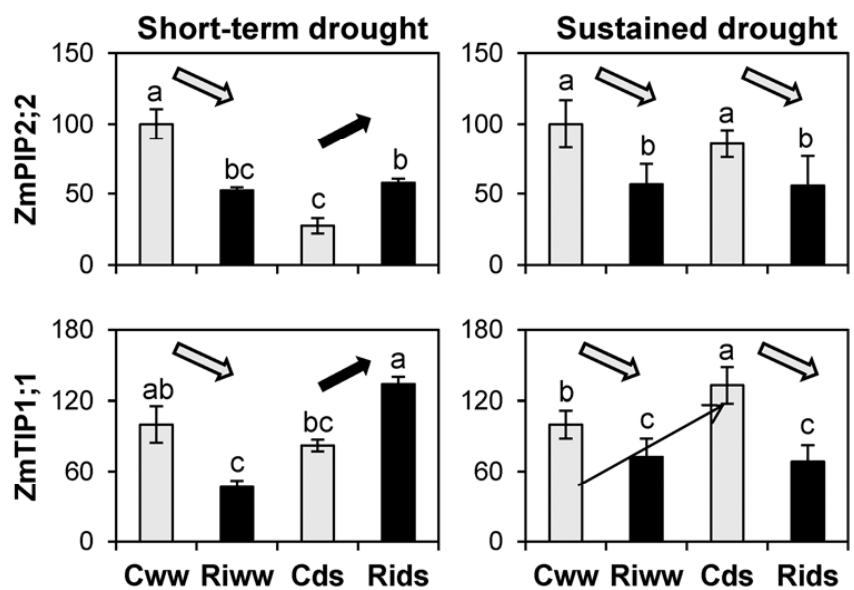

\section{PATTERN 5}
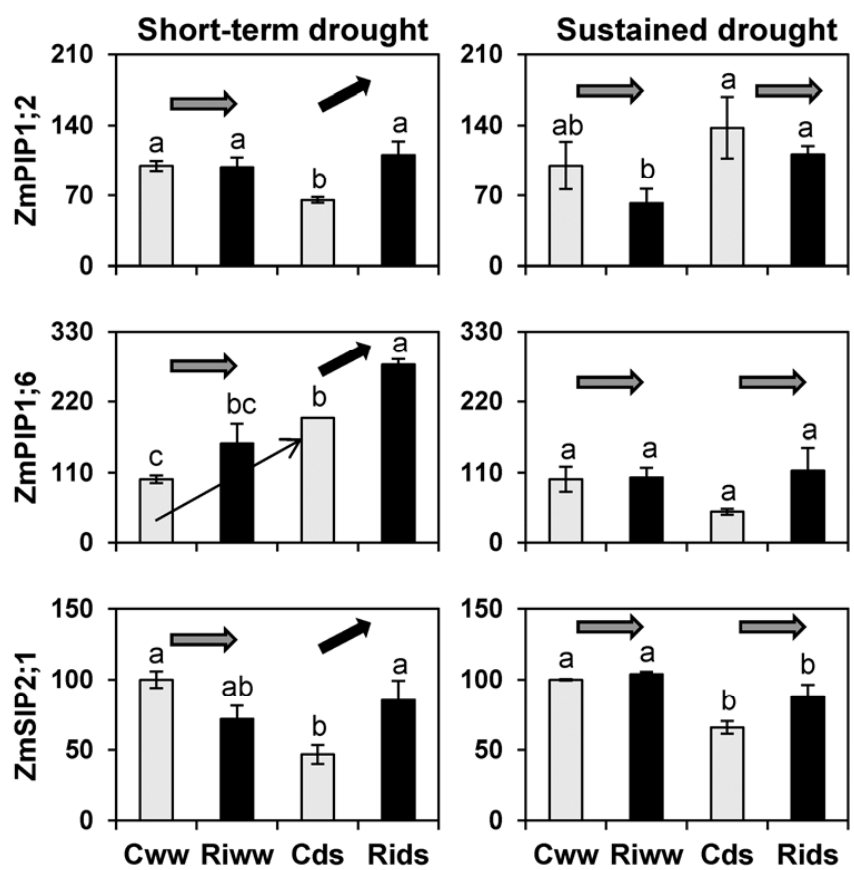

PIP2;4, and ZmTIP1;2 whereas, in AM plants, sustained drought stress enhanced ZmPIP2;5 accumulation and had no further effect on the rest of aquaporins.

\section{Functional characterization.}

Within each expression pattern group, the functional characterization of selected aquaporins was performed. The selection of the aquaporins was based on their high expression levels, the particular interest of their expression pattern, and the fact that they were not previously characterized by other authors.

Water transport. The water channel activity of ZmPIP1;3PIP1;4, ZmPIP2;2，ZmTIP1;1，ZmTIP1;2，ZmNIP1;1, ZmNIP2;1, and ZmNIP2;2 and co-expression of ZmPIP1;3 and ZmPIP2;2 was tested by heterologous expression in Xenopus laevis oocytes (Fig. 4).

All the tested aquaporins, except ZmPIP1;3-PIP1;4, facilitated water diffusion through the oocyte membrane. ZmPIP2;2 exhibited a high capacity for water transport, with osmotic permeability coefficient $\left(P_{f}\right)$ values similar to the positive control (ZmPIP2;5) (Chaumont et al. 2000). Injection of ZmPIP1;3$Z m P I P 1 ; 4$ cRNA did not induce any $P_{f}$ increase; however, we

\section{PATTERN 6}
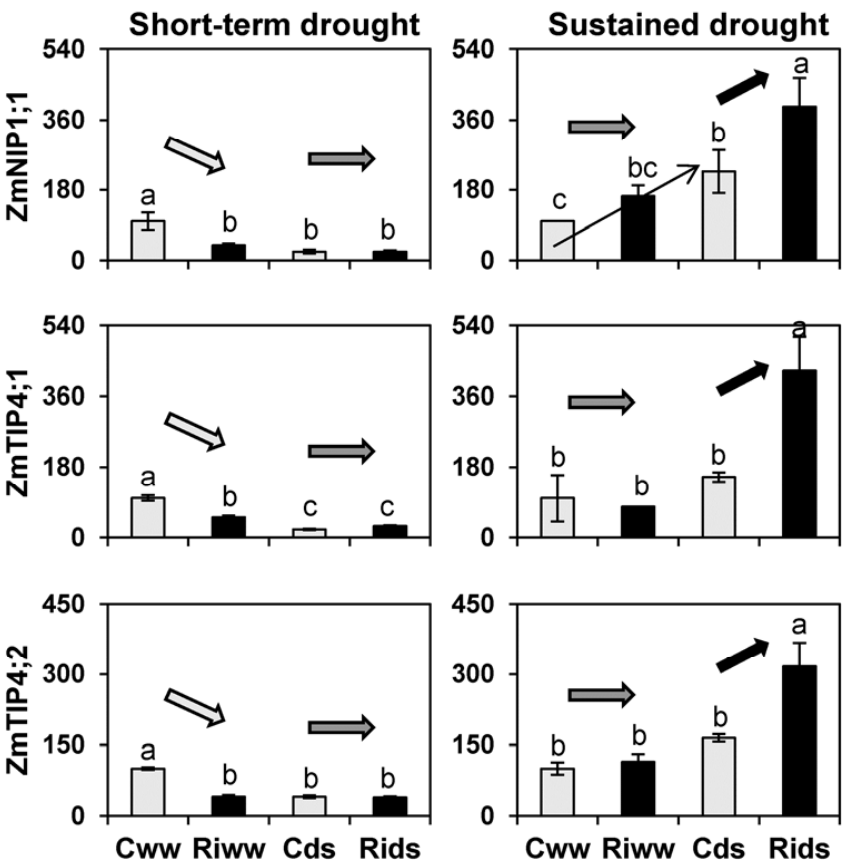

Fig. 2. Continued from previous page. 
evaluated the cooperative effect with PIP2 protein by coinjecting $Z m P I P 1 ; 3$ and $Z m P I P 2 ; 2$ cRNAs. When $0.5 \mathrm{ng}$ of $Z m P I P 2 ; 2$ cRNA was co-injected with $25 \mathrm{ng}$ of $Z m P I P 1 ; 3$ cRNA, a significant increase in $P_{f}$ was observed compared with oocytes injected with $0.5 \mathrm{ng}$ of $Z m P I P 2 ; 2 \mathrm{cRNA}$ alone, confirming that ZmPIP1 proteins need to be co-expressed with ZmPIP2s to detect their activity (Fetter et al. 2004).

Within the TIP subgroup, ZmTIP1;2 revealed water permeability, with a $P_{f}$ value of $0.9310^{-4} \mathrm{~m} \mathrm{~s}^{-1}$. For ZmTIP1;1 a $P_{f}$ value of $0.310^{-4} \mathrm{~m} \mathrm{~s}^{-1}$ was obtained.

All the NIPs analyzed seemed to increase the oocyte $P_{f}$ and, therefore, transport water, but at a low level. ZmNIP2;1 had $P_{f}$ values of approximately $0.2610^{-4} \mathrm{~m} \mathrm{~s}^{-1}$. ZmNIP1;1 and ZmNIP2;2 resulted in $P_{f}$ values of $0.1910^{-4} \mathrm{~m} \mathrm{~s}^{-1}$.

Glycerol transport. The glycerol channel activity of ZmPIP1;3-PIP1;4， ZmPIP2;2， ZmTIP1;1， ZmTIP1;2,
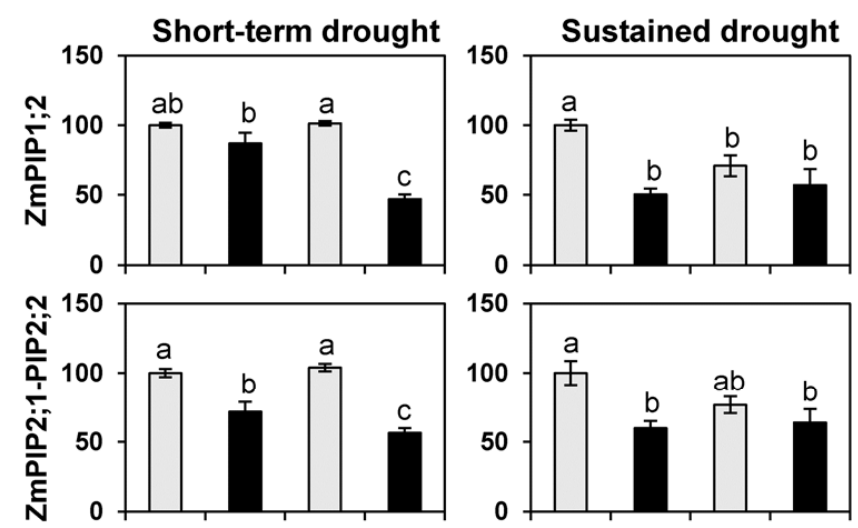

㐫
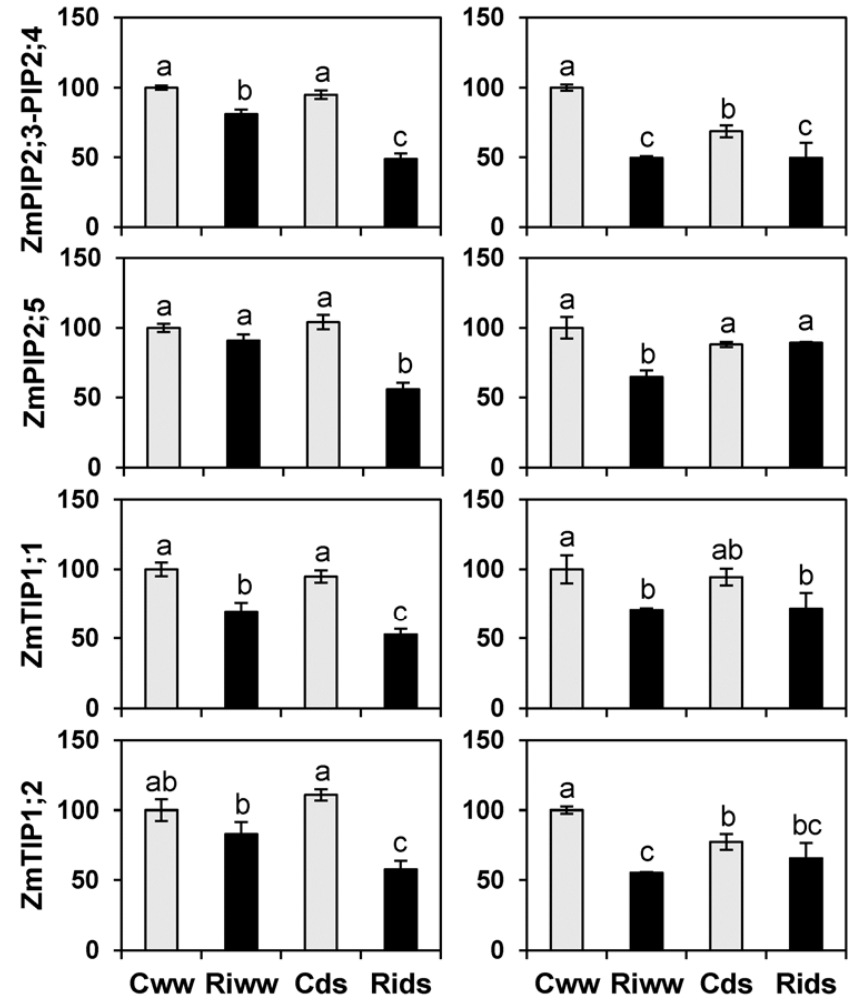

Fig. 3. Root protein accumulation patterns for ZmPIP1;2, ZmPIP2;1PIP2;2, ZmPIP2;3-PIP2;4, ZmPIP2;5, ZmTIP1;1, and ZmTIP1;2. C = uninoculated control, $\mathrm{Ri}=$ inoculated with Rhizophagus intraradices, $\mathrm{ww}=$ well-watered plants, and ds = plants subjected to drought stress. The mean and standard error for three independent protein quantifications are shown. Means followed by different letters are significantly different $(P<0.05)$ as determined by Duncan's multiple range and least significant difference tests.
ZmNIP1;1, and ZmNIP2;1 ZmNIP2;2 and co-expressed ZmPIP1;3 and ZmPIP2;2 was tested by heterologous expression in $X$. laevis oocytes. Only ZmNIP1;1 and ZmNIP2;1 showed the ability to transport glycerol in the oocyte system (Fig. 5).

Transport of nitrogen compounds. For testing urea, the yeast mutant YNVW1 was transformed with an empty vector or a vector containing rAQP9 (positive control), ZmPIP1;3ZmPIP1;4, ZmPIP2;2, ZmTIP1;1, ZmTIP1;2, ZmNIP1;1, or ZmNIP2;1 (Fig. 6). Yeast cells containing the empty vector were not able to grow on medium containing $1 \mathrm{mM}$ urea but cells expressing ZmTIP1;1 or ZmTIP1;2 grew on this medium, suggesting that they are able to transport urea. In accordance with recent findings by $\mathrm{Gu}$ and associates (2012), ZmNIP2;1 was also able to transport urea.

For testing ammonia, the yeast strain $\Delta$ mep1-3 was transformed with an empty vector or a vector containing $h A Q P 8$ as a positive control, ZmPIP1;3-PIP1;4, ZmPIP2;2, ZmTIP1;1, $Z m T I P 1 ; 2$, or ZmNIP1;1. None of the transformed yeasts was able to grow at $1 \mathrm{mM}$ ammonium ion $\left(\mathrm{NH}_{4}{ }^{+}\right)$supplied as a sole $\mathrm{N}$ source (Fig. 7). Yeast expressing the positive control, $\mathrm{ZmTIP} 1 ; 1$, or ZmTIP1;2 were able to grow at 2, 3, and $5 \mathrm{mM}$ $\mathrm{NH}_{4}^{+}$, indicating that they are permeable to $\mathrm{NH}_{3}$, whereas ZmPIP1;3-ZmPIP1;4, ZmPIP2;2, and ZmNIP1;1 are not.

Transport of the metalloids $B$ and Si. The yeast strain YNVW1 was transformed with an empty vector or a vector containing rAQP9, ZmPIP1;3-PIP1;4, ZmPIP2;2, ZmTIP1;1, $Z m T I P 1 ; 2, Z m N I P 1 ; 1$, or ZmNIP2;1 (Fig. 8). In the case of B, the measurement is based on the toxicity of boric acid for yeast growth.

Yeast transformants containing the empty vector or expressing ZmPIP1;3-PIP1;4 or ZmPIP2;2 were not affected by the increasing concentration of boric acid to $15 \mathrm{mM}$ in the growth medium, indicating no boric acid transport capacity of the respective aquaporin. At $5 \mathrm{mM}$ boric acid, growth inhibition due to toxicity appeared in transformants expressing the positive control rAQP9, ZmNIP2;1, ZmTIP1;1, or ZmTIP1;2 and continued to increase with the increase in supplied boric acid concentration. In the presence of $20 \mathrm{mM}$ boric acid, an almost total inhibition of growth of yeast strains expressing either of the two ZmTIP proteins was observed, indicating their high capacity to transport boric acid.

In the case of ZmNIP1;1, the growth inhibition was only observed above $10 \mathrm{mM}$ boric acid, suggesting a very low transport capacity. However, such boric acid transport capacity was not verified in the oocyte test system (Supplementary Fig. S3). In contrast, for ZmNIP2;1, and ZmTIP1;2, a boric acid transport capacity was detected in oocyte system.

To analyze the transport of silicic acid, Ge, a chemical analogue of $\mathrm{Si}$, was used (Ma and Yamaji 2008; Mitani et al. 2008). $\mathrm{GeO}_{2}$ is toxic to yeast when it is taken up (Tallberg et al. 2002). The yeast mutant YNVW1 was transformed with an empty vector or a vector containing $Z m P I P 1 ; 3-P I P 1 ; 4$, ZmPIP2;2, ZmTIP1;1, ZmTIP1;2, ZmNIP1;1, or ZmNIP2;1 as positive control, because it has been described as an $\mathrm{Si}$ transporter in both oocytes (Mitani et al. 2009) and the yeast system ( $\mathrm{Gu}$ et al. 2012). Apart from the positive control, none of the yeast transformants showed $\mathrm{GeO}_{2}$ toxicity in the tested concentration range (Fig. 9), indicating no transport of $\mathrm{Si}$.

$\mathrm{H}_{2} \mathrm{O}_{2}$ transport. The yeast strain YNVW1 was transformed with an empty vector or a vector containing $h A Q P 8$ (positive control), ZmPIP1;3-PIP1;4, ZmPIP2;2, ZmTIP1;1, ZmTIP1;2, ZmNIP1;1, or ZmNIP2; 1 (Fig. 10).

Because $\mathrm{H}_{2} \mathrm{O}_{2}$ is toxic for yeast cells, yeast expressing $\mathrm{H}_{2} \mathrm{O}_{2}$-permeable channels that can transport this compound does not grow on medium supplemented with this reactive oxygen species. As expected, the yeast mutant containing the 
empty vector grew at all tested $\mathrm{H}_{2} \mathrm{O}_{2}$ concentrations. The same result was found for ZmPIP1;3-ZmPIP1;4, indicating no transport capacity. Yeast cells expressing the positive control hAQP8 abolished its ability to grow above $0.2 \mathrm{mM} \mathrm{H}_{2} \mathrm{O}_{2}$ in the growth medium, like ZmTIP1;1 and ZmTIP1;2, indicating $\mathrm{H}_{2} \mathrm{O}_{2}$ transport capacity. Yeast expressing ZmPIP2;2, $\mathrm{ZmNIP} 1 ; 1$, or ZmNIP2;1 could not grow in the presence of 1.5 $\mathrm{mM} \mathrm{H} \mathrm{H}_{2} \mathrm{O}_{2}$, indicating a low capacity for $\mathrm{H}_{2} \mathrm{O}_{2}$ transport.

\section{DISCUSSION}

\section{Aquaporin regulation}

by the AM symbiosis and plant water relations.

In this study, both, short-term and sustained drought stress conditions caused a decrease in Jv and Lo parameters. With such decrease, plants restrict water movement along tissues and, ulti- mately, avoid water loss from roots to the soil (Aroca et al. 2007; Muhsin and Zwiazek 2002). Nevertheless, the response of AM plants differed from that of non-AM plants. It is known that AM symbiosis results in altered rates of water movement into, through, and out of the host plants (Augé 2001) and also modifies Jv and Lo parameters (Aroca et al. 2007; Ruiz-Lozano et al. 2009).

Aquaporins provide a low resistance pathway for the movement of water across a membrane. Furthermore, because aquaporins can be gated, this provides greater control for the movement of water along plant tissues. PIP and TIP isoforms have been recognized as central pathways for transcellular and intracellular water transport (Maurel et al. 2008). Thus, it seems likely that mycorrhizal symbiosis causes significant changes in aquaporin activity of host plants (Aroca et al. 2007; Porcel et al. 2006; Ruiz-Lozano et al. 2009; Uehlein et al. 2007) and

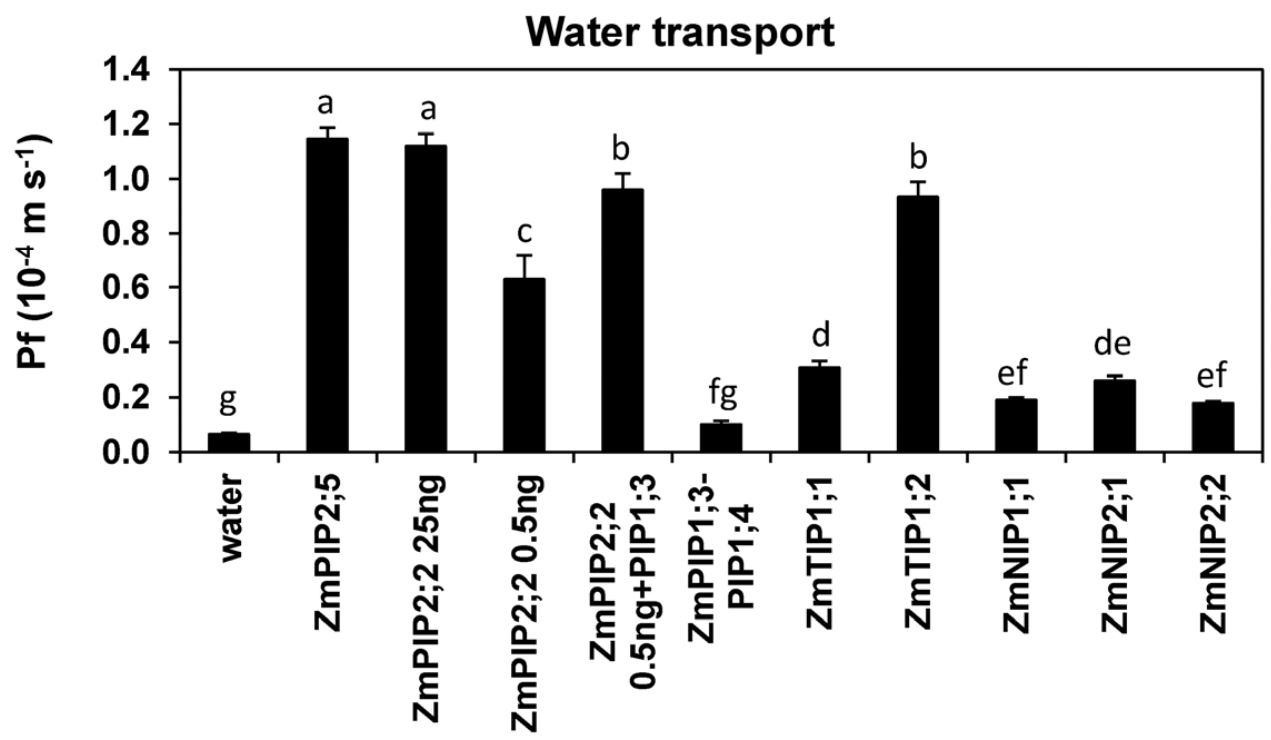

Fig. 4. Water transport assay in Xenopus laevis oocytes expressing $25 \mathrm{ng}$ of ZmPIP2;5 as a positive control; 25 or $0.5 \mathrm{ng}$ of ZmPIP2;2; coexpression of ZmPIP1;3; and $0.5 \mathrm{ng}$ of ZmPIP2;2 or $25 \mathrm{ng}$ of ZmPIP1;3-PIP1;4, ZmTIP1;1, ZmTIP1;2, ZmNIP1;1, ZmNIP2;1, and ZmNIP2;2. Water injection was used as negative control. Values correspond to mean water permeabilities (Pf). The number of oocytes measured for each protein was 10 to 20 . Means followed by different letters are significantly different $(P<0.05)$ as determined by Duncan's multiple range and least significant difference tests.

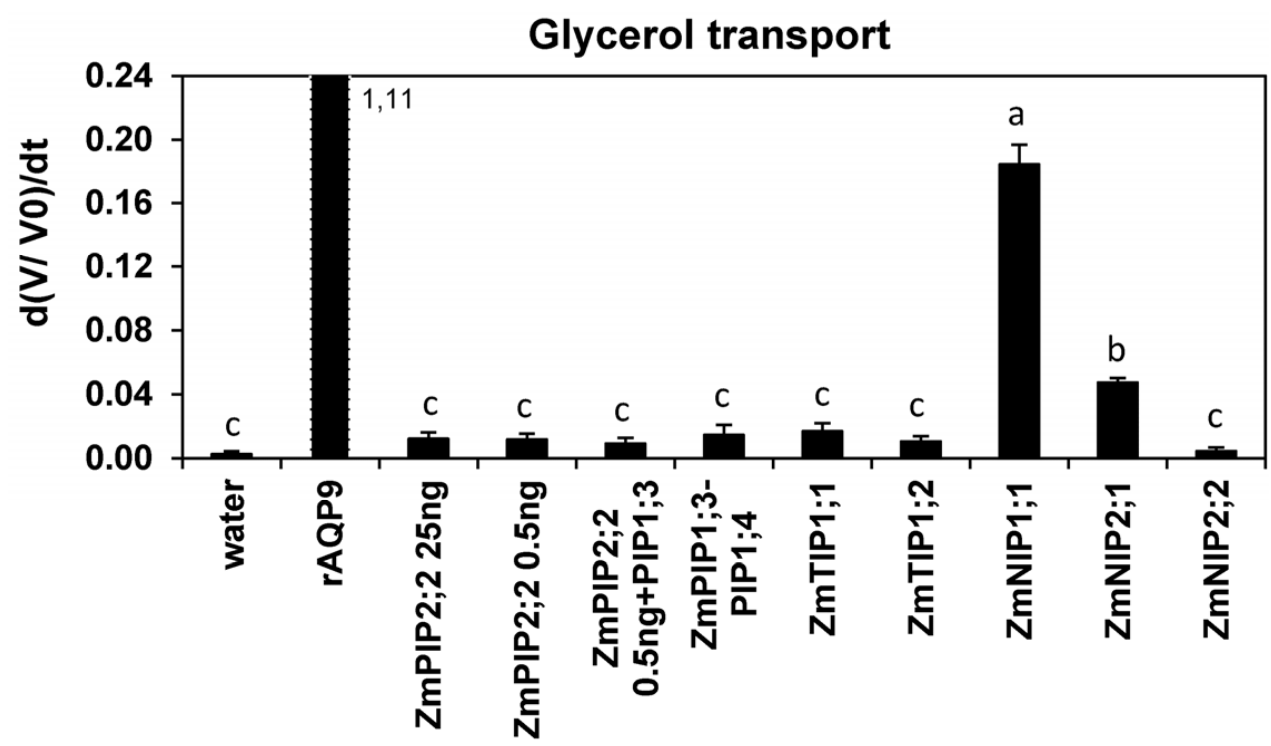

Fig. 5. Glycerol transport assay in Xenopus laevis oocytes expressing $25 \mathrm{ng}$ of rAQP9 as a positive control; 25 or $0.5 \mathrm{ng}$ of ZmPIP2;2; coexpression of ZmPIP1;3; and $0.5 \mathrm{ng}$ of ZmPIP2;2 or $25 \mathrm{ng}$ of ZmPIP1;3-PIP1;4, ZmTIP1;1, ZmTIP1;2, ZmNIP1;1, ZmNIP2;1, and ZmNIP2;2. Water injection was used as negative control. Values are mean of the time-course volume changes $[\mathrm{d}(\mathrm{V} / \mathrm{V} 0) / \mathrm{dt}]$. Means followed by different letters are significantly different $(P<$ 0.05 ) as determined by Duncan's multiple range and least significant difference tests. 
some of the plant aquaporins might be important for these mycorrhizal responses.

All the PIP aquaporins analyzed in this study have typical features of water transporting aquaporins (Sui et al. 2001; Wallace et al. 2002; Forrest and Bhave 2007). Indeed, all PIP2s transported water and the expression of ZmPIP2;2 in oocytes resulted in a particularly high membrane water permeability, especially when co-expressed with PIP1s. Therefore, regulation of PIP aquaporins was expected to be a key point in regulation of plant water transport by AM symbiosis.

Under short-term drought stress conditions, AM plants maintained higher $\mathrm{Jv}$ and Lo values than non-AM plants. Under sustained drought stress, the soil water resources were highly reduced and AM plants also decreased their Jv and Lo values. Such different behavior under the two different stress conditions correlated with the expression patterns of PIP proteins regulated by AM. Under short-term drought stress, almost all PIP proteins

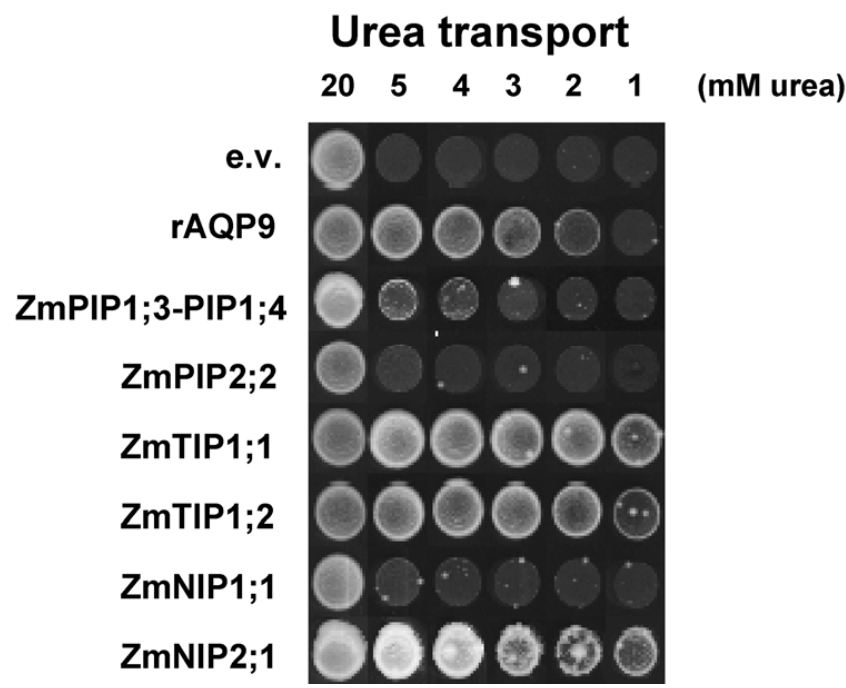

Fig. 6. Urea transport assay in YNVW1 yeast strain transformed with the empty vector (negative control), rAQP9 (positive control), ZmPIP1;3ZmPIP1;4, ZmPIP2;2, ZmTIP1;1, ZmTIP1;2, ZmNIP1;1, or ZmNIP2;1. Yeasts were spotted on medium containing various concentrations of urea as sole nitrogen source and growth was recorded after 9 days at $28^{\circ} \mathrm{C}$. All data were duplicated in at least two independent experiments with consistent results.

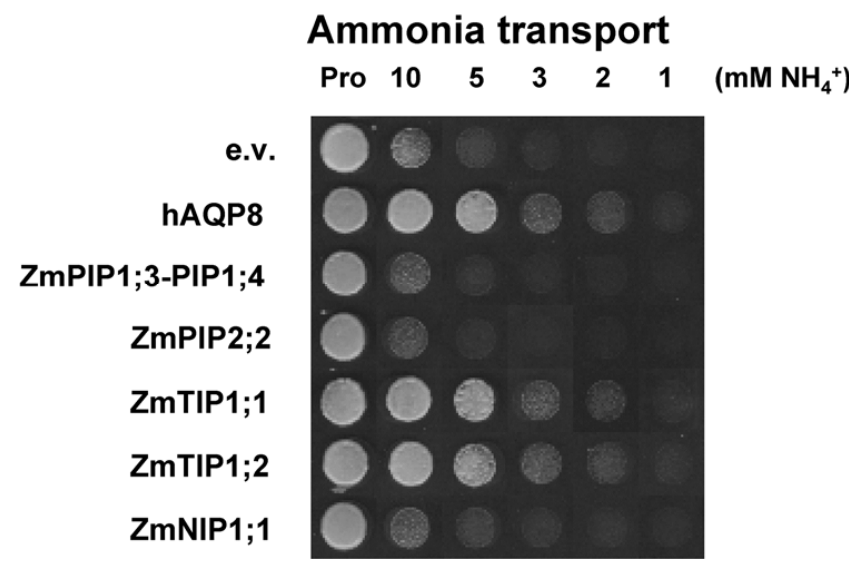

Fig. 7. Ammonia transport assay in $\triangle$ mep1-3 yeast strain transformed with the empty vector (negative control), hAQP8 (positive control), ZmPIP1;3PIP1;4, ZmPIP2;2, ZmTIP1;1, ZmTIP1;2 or ZmNIP1;1. Yeasts were spotted on medium containing proline or various concentrations of ammonia as sole nitrogen sources and growth was recorded after 9 days at $28^{\circ} \mathrm{C}$. All data were duplicated in at least two independent experiments with consistent results. were downregulated in non-AM plants whereas, in AM plants, the expression of most PIP proteins was kept high or even increased (ZmPIP1;1, ZmPIP1;2, ZmPIP1;3, ZmPIP1;4, $\mathrm{ZmPIP1}$;6, ZmPIP2;2, and ZmPIP2;4), correlating with the measured Jv and Lo. In experiment 2, we observed a downregulation by $\mathrm{AM}$ of an important number of PIP genes (including most of those selected in this study: ZmPIP1;1, ZmPIP1;3/ZmPIP1;4, ZmPIP2;2, and ZmPIP2;4) under both well-watered and sustained drought conditions, probably as a mechanism to prevent water loss (Porcel et al. 2006). Such downregulation and the decrease of protein accumulation after mycorrhization correlated with the lower $\mathrm{Jv}$ and Lo in AM plants.

Under sustained drought conditions, Lh was maintained at high levels in AM plants. These effects have been related to the increased absorbing surface caused by soil-growing hyphae, combined with the fungal capability to take up water from soil pores inaccessible to roots, because AM hyphae represent a lowresistance pathway to root cells (Allen 2007, 2009; Lehto and Zwiazek 2011; Ruiz-Lozano 2003). Hyphae have also been suggested to maintain liquid continuity in the soil and limit the loss of soil hydraulic conductance caused by air gaps (Smith et al. 2010). Thus, water movement through AM fungal hyphae under such conditions can be critical to improve the water supply to the plant and, therefore, cell-to-cell and apoplastic pathways

\section{Boric acid transport}

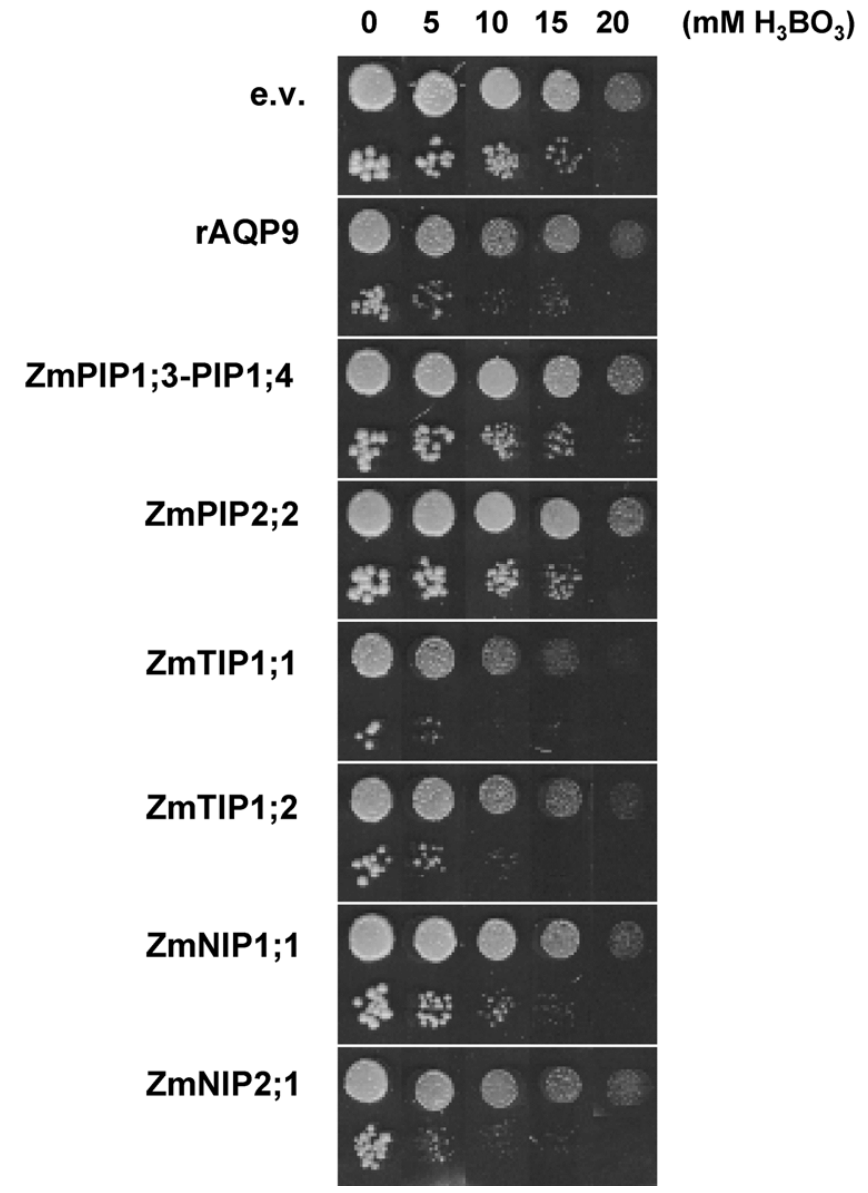

Fig. 8. Boric acid transport assay by a yeast growth and survival test on medium containing boric acid. YNVW1 yeast strain was transformed with the empty vector (negative control), rAQP9 (positive control), ZmNIP2;1, ZmPIP1;3-PIP1;4, ZmPIP2;2, ZmTIP1;1, ZmTIP1;2, or ZmNIP1;1. Yeasts were spotted on medium containing various concentrations of boric acid and growth was recorded after 6 days at $28^{\circ} \mathrm{C}$. All data were duplicated in at least two independent experiments with consistent results. 
increase (Barzana et al. 2012). On the other hand, AM fungal aquaporins have been related to water transport in the extraradical mycelium and in the periarbuscular membrane ( $\mathrm{Li}$ et al. 2013). Thus, in AM plants, the enhanced Lo values under shortterm drought stress and Lh values under sustained drought could be due to the activity of the fungal aquaporins.

Apart from the clear correlations between root hydraulic parameters and aquaporin expression, there are also some uncorrelations. The high values of $\mathrm{Jv}$ and Lo in experiment 1 under well-watered conditions contrast with changes in the expression or protein accumulation of the different analyzed aquaporins (except for ZmPIP2;5). This lack of correlation has already been observed (Aroca et al. 2007; Boursiac et al. 2005; Ruiz-Lozano et al. 2009) and may be due to the fact that aquaporin regulation is not only restricted to the transcriptional level but also subjected to post-transcriptional modifications. In addition, aquaporins are not a unique way to control Lo. Symplastic movement of water via plasmodesmata may contribute significantly to Lo, depending on the exact environmental circumstances (Galmés et al. 2007). In this regard, Blee and Anderson (1998) found that plasmodesmata were increased in the surroundings of arbusculated cells, which may well assist symplastic water movement. Moreover, a compensatory mechanism among AM fungal aquaporins and host plant aquaporins has been proposed (Aroca et al. 2009). Thus, the downregulation of host plant aquaporins could be compensated with the activity of fungal aquaporins to maintain high $\mathrm{Jv}$ and Lo values in AM roots under well-watered conditions. Also, the amount of ZmPIP2;5 protein was maintained high in these plants, and ZmPIP2;5 is one of the major aquaporins in maize roots (Hachez et al. 2006).

In experiment 2 , drought itself had little effect on the transcription of PIP genes or the accumulation of PIP proteins but had a significant impact on $\mathrm{JV}$ and Lo, decreasing both parameters. The maintenance of PIP gene expression under these conditions may partially compensate for the reduction of Lo, as has been shown in olive (Lovisolo et al. 2007). On the other hand, the posttranscriptional regulation may have an important role under drought. In fact, there is evidence of a relationship between the reactive oxygen species produced under abiotic stress and the internalization and changes in the

\section{$\mathrm{GeO}_{2}$ transport}

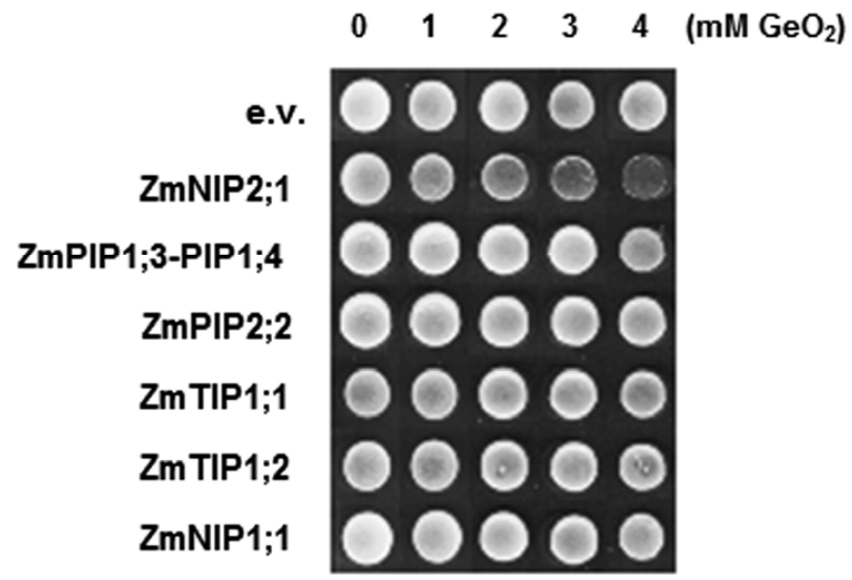

Fig. 9. Silicon (Si) transport assay by a yeast growth and survival test on medium containing $\mathrm{GeO}_{2}$ as an analogue of Si. YNVW1 yeast strain was transformed with the empty vector (negative control), ZmNIP2;1 (positive control), ZmPIP1;3-PIP1;4, ZmPIP2;2, ZmTIP1;1, ZmTIP1;2, or $\mathrm{ZmNIP} 1 ; 1$. Yeasts were spotted on medium containing various concentrations of $\mathrm{GeO}_{2}$ and growth was recorded after 6 days at $28^{\circ} \mathrm{C}$. All data were duplicated in at least two independent experiments with consistent results. subcellular localization of PIP proteins (Boursiac et al. 2008) or with a direct blockage of aquaporins (Ye and Steudle 2006). This would lead to a decrease in Lo without changing the expression or accumulation of these aquaporins (Benabdellah et al. 2009).

In this study, the expression pattern for some of the analyzed TIP proteins was of interest. ZmTIP1;1 and ZmTIP1;2 are structurally able to transport water in high amounts, as has been shown for many TIP isoforms in different plant species (Gerbeau et al. 1999; Vera-Estrella et al. 2004). TIP proteins are the most abundant aquaporins in plants and are mainly associated with the tonoplast (Hunter et al. 2007). It has been proposed that TIP proteins may provide a quick way for cellular osmotic balance by controlling the exchange of water between vacuole and cytosol (Forrest and Bhave 2007), playing an important role under osmotic stress conditions (Katsuhara et al. 2008; Vera-Estrella et al. 2004). Thus, TIP proteins may also have an influence on $\mathrm{Jv}$ and Lo by affecting exchanges of water between transcellular and symplastic water pathways. In our study, ZmTIP1;1 and ZmTIP1;2 were highly expressed in all treatments and, in the oocyte system, both exhibited a high capacity for water transport. Mycorrhization upregulated their expression under short-term drought stress but, under sustained drought, they were downregulated, correlating with Jv and Lo parameters. However, in the case of ZmTIP 1;1, its upregulation in non-AM plants under sustained drought and the maintenance of its protein content is contradictory to the decrease of $\mathrm{Jv}$ and Lo, which suggest that its specific function may not be the transport of water; however, this remains to be elucidated.

\section{Aquaporin characterization for the transport} of solutes of physiological importance for the plant.

It has been proposed that the role of aquaporins in AM symbiosis could be more complex than simply regulating plant water status (Maurel and Plassard 2011). Uehlein and associates (2007) described induction by the AM symbiosis of specific PIP and NIP isoforms exhibiting permeability to water and to $\mathrm{NH}_{3}$, respectively. Authors suggested that these aquaporins could be involved in the symbiotic exchange processes between the fungus and the plant. Our results point in the same direction, because many of the aquaporin genes regulated by the AM symbiosis can transport a variety of compounds of physiological importance for the plant.

\section{Hydrogen peroxide transport}

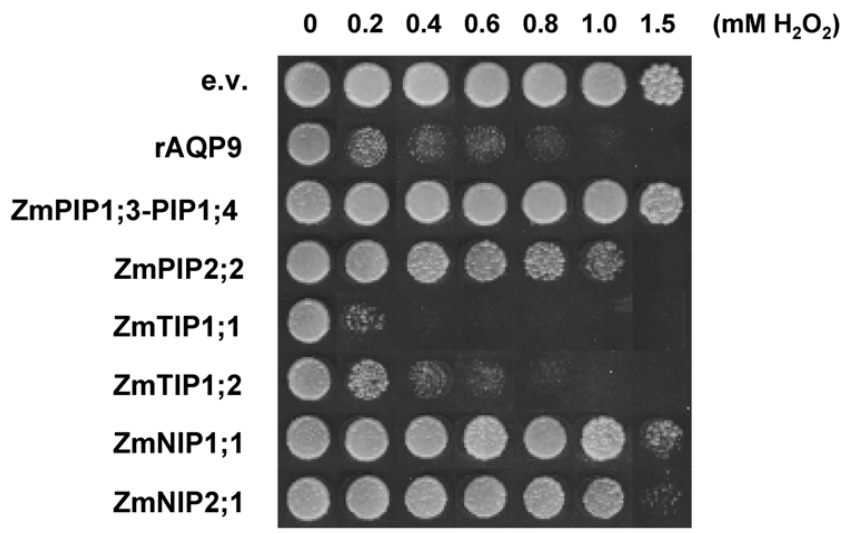

Fig. 10. $\mathrm{H}_{2} \mathrm{O}_{2}$ transport assay by a yeast growth and survival test on medium containing $\mathrm{H}_{2} \mathrm{O}_{2}$. YNVW1 yeast strain transformed with the empty vector (negative control), hAQP8 (positive control), ZmPIP1;3-PIP1;4, ZmPIP2;2, ZmTIP1;1, ZmTIP1;2, ZmNIP1;1, or ZmNIP2;1 were spotted on agar plates containing various concentrations of $\mathrm{H}_{2} \mathrm{O}_{2}$. Growth was recorded after 6 days at $28^{\circ} \mathrm{C}$. All data were duplicated in at least two independent experiments with consistent results. 
Transport of glycerol. The utilization of glycerol is well known in fungi and bacteria but not in plants (Dietz et al. 2011). Thus, despite the numbers of aquaporins that have been described as glycerol transporters in plants, the physiological implication of this molecule remains unclear. Like other compatible solutes, glycerol must be able to come into the cells and move through the lipids bilayer very fast to respond to osmotic imbalances. Some aquaporins from plants have been characterized as functional glycerol transporters. Most of them belong to the NIP subgroup (Cabello-Hurtado and Ramos 2004; Weig and Jakob 2000). NIPs are supposed to originally come from bacteria by horizontal gene transfer (Zardoya et al. 2002) and were later adapted for glycerol transport, suggesting that this was a selective advantage for plants (Gustavsson et al. 2005). We demonstrated in oocytes that ZmNIP1;1 and ZmNIP2;1 can transport glycerol. ZmNIP1;1 revealed a high transport capacity for glycerol, being also able to facilitate the flux of water and $\mathrm{H}_{2} \mathrm{O}_{2}$. We have shown that $\mathrm{ZmNIP1;1}$ is regulated in a manner different from most of the other aquaporins, increasing its expression under sustained drought stress in non-AM plants and further enhancing its expression in AM plants. In addition, under well-watered conditions, the AM symbiosis affects ZmNIP1;1 in a different way in both experiments, downregulating its expression in experiment 1 but not affecting its expression in experiment 2 . This effect was observed only in aquaporins grouped in pattern $6(\mathrm{ZmNIP1} 1$, ZmTIP4; 1, and ZmTIP4;2) and for ZmTIP1;2 (pattern 2), indicating that these aquaporins are finely regulated by the AM symbiosis and that the regulation goes beyond water conditions.

The transport of glycerol has also been suggested for TIP4 isoforms, because their selectivity filter contains amino acids of small size that increase the pore diameter (Bansal and Sankararamakrishnan 2007), allowing the possible transport of big molecules such as glycerol. In addition, their selectivity filter is not adequate for water transport (Bansal and Sankararamakrishnan 2007; Wallace and Roberts 2004), which suggests that aquaporins grouped in pattern 6 may fulfil specific functions in the transport of solutes, including glycerol, that could be important for the AM symbiosis or for the plant-fungus interaction (Dietz et al. 2011) under sustained drought stress conditions. Indeed, a study has shown a transfer of glycerol from host plant to pathogenic fungi (Wei et al. 2004), and Gustavsson and associates (2005) suggested that exporting plant glycerol may be also important for symbiotic fungi and that this could be the reason why glycerol transporters were fixed in plant genomes. This would explain why ZmNIP1;1, ZmTIP4;1, and ZmTIP4;2 (pattern 6) are so finely regulated by the AM symbiosis and are the sole aquaporins upregulated in AM plants under sustained drought conditions compared with non-AM plants.

Transport of nitrogen compounds. Nitrogen is one of the most important nutrients for all living organisms, being needed for the synthesis of compounds essential for growth processes. $\mathrm{NH}_{4}{ }^{+}$and its conjugated base $\mathrm{NH}_{3}$ are the potential primary sources of $\mathrm{N}$ in plant nutrition. In the AM symbiosis, $\mathrm{NH}_{4}{ }^{+}$is suggested to be the major nitrogen compound transferred to the host plant, with urea playing a role as an intermediate solute (Govindarajulu et al. 2005; Perez-Tienda et al. 2011; Tian et al. 2010), which could be also involved in fungus-based nitrogen nutrition of plant hosts (Dietz et al. 2011).

Aquaporins have been pointed out as low-affinity channels for $\mathrm{N}$ absorption, mobilization, and detoxification in the nitrogen metabolism in plants (Liu et al. 2003b) and in the AM symbiosis (Pérez-Tienda et al. 2011; Uehlein et al. 2007). In maize, most of the TIP proteins possess an ar/ $\mathrm{R}$ (aromatic/arginine) region that enables the protein to transport $\mathrm{NH}_{3}$ and urea (Jahn et al. 2004; Sui et al. 2001). Indeed, several TIP proteins have been shown to transport these compounds (Jahn et al.
2004; Liu et al. 2003b; Loque et al. 2005), including ZmTIP1;1 and ZmTIP1;2, characterized in this study.

Transport of urea and $\mathrm{NH}_{3}$ or $\mathrm{NH}_{4}{ }^{+}$into the vacuole would avoid their toxicity in the cytoplasm or allow storage of $\mathrm{N}$ (Wang et al. 2008) and, whenever required as an $\mathrm{N}$ source, these compounds could be remobilized by a passive, lowaffinity transport pathway, such as that provided by TIP proteins (Liu et al. 2003b). In this study, the expression of most of the aquaporins decreased in non-AM plants under short-term drought stress conditions whereas, in AM plants, the expression of several aquaporin genes was maintained under such conditions. ZmTIP1;1 and ZmTIP1;2 were upregulated in AM plants compared with non-AM ones. Such upregulation would promote the transport not only of water but also of nitrogen compounds (Uehlein et al. 2007). Under sustained drought stress, the expression of most aquaporins and their protein accumulation decreased in non-AM plants, probably because of a lower availability of mineral nutrients for the plant.

Transport of metalloids. B and $\mathrm{Si}$ are categorized as metalloids. At neutral $\mathrm{pH}$, both are present in soil solution mainly as noncharged molecules (Miwa et al. 2009) and are not metabolized by plants. They are transported as boric and silicic acids (Miwa et al. 2009). High-affinity transporter systems (BOR1 for $\mathrm{B}$ and $\mathrm{Lsi} 2$ for $\mathrm{Si}$ ) generate a concentration gradient from the soil to the root cells, allowing a passive transport of both nutrients through aquaporins (Mitani et al. 2009). Both metalloids have key structural functions in plant cells: B cross-links with the pectin fraction of cell walls and polymers of hydrated silica gel are important for the physical strength of plant cells, especially in monocots such as maize (Miwa et al. 2009).

Maize has a low B requirement to maintain normal vegetative growth. However, at the reproductive stage, maize needs as much B as other species (Blevins and Lukaszewski 1998). At high concentrations, $\mathrm{B}$ is toxic, which is why maize must tightly control $\mathrm{B}$ distribution to maintain adequate $\mathrm{B}$ levels in their cells (Miwa et al. 2009).

Under sustained drought, reduced plant transpiration can lead to B deficiencies, which can affect negatively plant growth. Thus, the B requirements in non-AM plants may be guaranteed by the aquaporins that can transport B (ZmTIP1;1, ZmTIP1;2, $\mathrm{ZmNIP1}$; , and ZmNIP2;2), which are highly expressed. However, we observed that the mycorrhization decreased the expression of almost all the aquaporins that can transport B. This can be due to the fact that the AM fungus may provide B directly to the host plant (Lehto et al. 2010), and plant aquaporins involved in B transport are downregulated in order to avoid toxicity due to an excess of B.

The case of $\mathrm{Si}$ is different because it is actively absorbed and accumulates in high amounts in maize tissues, where it functions in tolerance to abiotic stresses (Ma and Yamaji 2006, 2008). Thus, the existence of several mechanisms of transport and regulation of $\mathrm{Si}$ uptake is expected in maize. Both ZmNIP2;1 and ZmNIP2;2 were characterized by Mitani and associates (2009) as Si transporters.

In the two experiments described here, mycorrhization reduced the expression of ZmNIP2;1 and ZmNIP2;2 under wellwatered conditions and the expression was kept low under drought stress conditions. The most direct explanation for this effect is that Si deposited beneath the cuticle can mechanically impede penetration by fungi and, thereby, a diminution of $\mathrm{Si}$ uptake in mycorrhizal plants can be expected. Even more, soluble $\mathrm{Si}$ acts as a modulator of host resistance to fungal infection and colonization (Fauteux et al. 2005), suggesting that soluble $\mathrm{Si}$ should be also reduced in mycorrhizal plants.

Transport of $\mathrm{H}_{2} \mathrm{O}_{2}$. At low concentrations, $\mathrm{H}_{2} \mathrm{O}_{2}$ acts as a signal molecule controlling different essential processes in plants during normal growth and development (Bienert et al. 
2006; Quan et al. 2008) but it also functions as a defensive signal molecule against various abiotic and biotic stresses (Bienert et al. 2006; Miller et al. 2010). On the other hand, as an oxidant molecule, it react with various cellular targets causing cell damage and, at high concentrations, it orchestrates programmed cell death (Bienert et al. 2006; Quan et al. 2008). Because of these opposing functions, maintaining the level of $\mathrm{H}_{2} \mathrm{O}_{2}$ in a normal dynamic balance is used by plants to activate stress responses that help the plant cope with environmental changes (Quan et al. 2008). $\mathrm{H}_{2} \mathrm{O}_{2}$ has almost the same size, electrochemical properties, and capacity to form hydrogen bonds as does water (Bienert et al. 2007). Indeed, some plant aquaporins were shown to transport $\mathrm{H}_{2} \mathrm{O}_{2}$ (Bienert et al. 2007; Dynowski et al. 2008) and, in this study, all aquaporins able to transport water could also transport $\mathrm{H}_{2} \mathrm{O}_{2}$, especially ZmTIP1;1. Bienert and associates $(2006,2007)$ proposed that TIP1s could play a key role in the detoxification of excess $\mathrm{H}_{2} \mathrm{O}_{2}$ generated under stress conditions. This idea fits with the high gene expression and protein content maintained for ZmTIP1;1 under both short-term and sustained drought stress conditions.

\section{Conclusion.}

This study shows that the AM symbiosis regulates the expression of a wide number of aquaporin genes in the host plant, comprising members of the different aquaporin subfamilies. Most of these aquaporins can transport water and also other molecules of physiological importance for plant performance. The regulation of these genes depends on the watering conditions and on the severity of the drought stress imposed. Indeed, under short-term drought, the AM symbiosis upregulated the aquaporins grouped in patterns $2,3,4$, and 5, and only those from patterns 1 and 6 were kept unaffected. In contrast, when the drought was sustained, the AM symbiosis downregulated the aquaporins grouped in patterns 1,2 , and 4, maintained unaffected those from patterns 3 and 5, and upregulated only the aquaporins from pattern 6 . This suggests that, under short-term drought conditions, the AM symbiosis can stimulate further the physiological processes in which these aquaporins could be involved, maybe because these plants have access to water resources which are inaccessible to non-AM plants. In contrast, when the drought becomes sustained, the AM symbiosis restricts most of the processes in which these aquaporins may participate. In non-AM plants, regardless of drought stress intensity, the response is a general downregulation of aquaporins and of the physiological processes related to these aquaporins. Thus, we propose that, in addition to the direct activity of AM fungal hyphae in taking up water and nutrients for the host plant, the AM symbiosis acts on the host plant aquaporins in a concerted manner to alter plant water relations and its physiology and allow it to cope better with stressful environmental conditions. In support of this idea, we found that AM plants exhibited higher Lo and Lh under short-term and sustained drought, respectively. Moreover, apart from the plant growth enhancement under wellwatered conditions, AM plants were still able to grow more than non-AM plants under short-term and under sustained drought conditions ( 35 and $26 \%$, respectively). In any case, future studies should be devoted to elucidating the specific function of each aquaporin isoform regulated by the AM symbiosis in order to shed further light on exactly how the symbiosis alters plant fitness under stressful conditions.

\section{MATERIALS AND METHODS}

This study consisted of two independent experiments with the same experimental design but with a different soil substrate and a different duration and application of the drought stress treatments.

\section{Experimental design and statistical analysis.}

The two experiments consisted of a factorial design with two factors: i) inoculation treatment, with noninoculated control plants and plants inoculated with the AM fungus Rhizophagus intraradices strain EEZ 58; and ii) water regime, so that one half of the plants were cultivated under well-watered conditions throughout the entire experiment and the other half of the plants were subjected to drought stress. In the first experiment plants were subjected to a short-term drought stress for 4 days whereas, in the second experiment, plants were subjected to a sustained drought stress for 12 days. Both experiments had a total duration of 8 weeks. Each treatment had 10 replicates, giving a total of 40 pots for each experiment. Each experiment was repeated at least twice.

Within each drought experiment, data were subjected to analysis of variance with inoculation treatment, water regime, and inoculation treatment-water regime interaction as sources of variation. Post hoc comparisons with the Duncan and least significant difference tests were used to find out differences between groups.

\section{Soil and biological materials.}

In experiment 1 , loamy soil was collected from the ground at the Zaidin Experimental Station (Granada, Spain). The soil had a $\mathrm{pH}$ of 8.1 (measured in water, 1:5 [wt/vol]); $1.5 \%$ organic matter; and nutrient concentrations of $\mathrm{N}$ at $1.9 \mathrm{~g} \mathrm{~kg}^{-1}$, $\mathrm{P}\left(\mathrm{NaHCO}_{3}\right.$-extractable $\left.\mathrm{P}\right)$ at $1 \mathrm{~g} \mathrm{~kg}^{-1}$, and $\mathrm{K}$ at $6.9 \mathrm{~g} \mathrm{~kg}^{-1}$.

In experiment 2, loamy soil was collected from Dúrcal (Granada, Spain). The soil had a $\mathrm{pH}$ of 8.2 (measured in water, 1:5 [wt/vol]); $1.8 \%$ organic matter; and nutrient concentrations of $\mathrm{N}$ at $2.5 \mathrm{~g} \mathrm{~kg}^{-1}, \mathrm{P}\left(\mathrm{NaHCO}_{3}\right.$-extractable $\left.\mathrm{P}\right)$ at $6.2 \mathrm{~g} \mathrm{~kg}^{-1}$, and $\mathrm{K}$ at $13.2 \mathrm{~g} \mathrm{~kg}^{-1}$.

Both soils were sieved $(5 \mathrm{~mm})$, diluted with quartz-sand $(<2$ $\mathrm{mm} ; 1: 1$, soil/sand [vol/vol]), and sterilized by steaming $\left(100^{\circ} \mathrm{C}\right.$ for $1 \mathrm{~h}$ on three consecutive days).

Two seeds of maize (Z. mays L. 'Potro') were sown in pots containing $1 \mathrm{~kg}$ of the respective soil/sand mixture as described above, and thinned to one seedling per pot after emergence.

Mycorrhizal inoculum was bulked in an open-pot culture of $Z$. mays L. and consisted of soil, spores, mycelia, and infected root fragments. The AM fungus was $R$. intraradices (Schenck and Smith), strain EEZ 58. Inoculum (10 g) with approximately 60 infective propagules per gram (according to the most probable number test) was added to appropriate pots at sowing time. Noninoculated control plants received the same amount of autoclaved mycorrhizal inoculum together with a 3-ml aliquot of a filtrate $(<20 \mu \mathrm{m})$ of the AM inoculum in order to provide a general microbial population free of AM propagules.

\section{Growth conditions.}

The experiments were carried out under greenhouse conditions with temperatures of 19 to $25^{\circ} \mathrm{C}$; light and darkness of 16 and $8 \mathrm{~h}$, respectively; relative humidity of 50 to $60 \%$; and an average photosynthetic photon flux density of $800 \mu \mathrm{mol} \mathrm{m} \mathrm{m}^{-2} \mathrm{~s}^{-1}$, as measured with a light meter (model LI-188B; LICOR, Lincoln, NE, U.S.A.). Plants were cultivated for a total of 8 weeks.

Short-term drought stress. Soil moisture was measured with a ML2 ThetaProbe (AT Delta-T Devices Ltd., Cambridge, U.K.), as previously described (Porcel and Ruiz-Lozano 2004). Water was supplied daily to maintain soil at $100 \%$ of field capacity during the first 52 days after sowing. Then, half of the plants were allowed to dry by withholding water irrigation for 4 days, while the other half was maintained at field capacity. After these 4 days, plants were harvested.

Sustained drought stress. Soil moisture was measured with the ML2 ThetaProbe (AT Delta-T Devices Ltd.). Water was supplied daily to maintain soil at $100 \%$ of field capacity during 
the first 6 weeks after sowing. Then, half of the plants were allowed to dry until soil water content reached $55 \%$ of field capacity ( 2 days needed), while the other half were maintained at field capacity. The soil water content was measured daily with the ThetaProbe ML2 before rewatering (at the end of the afternoon), reaching a minimum soil water content approximately $50 \%$ of field capacity. The amount of water lost was added to each pot in order to keep the soil water content at the desired levels (Porcel and Ruiz-Lozano 2004). Plants were maintained under such conditions for 10 additional days before harvesting.

\section{Physiological measurements.}

Biomass production. At harvest ( 8 weeks after sowing), the shoot and root system of six replicates per treatment were separated and the shoot dry weight measured after drying in a forced-hot-air oven at $70^{\circ} \mathrm{C}$ for 2 days.

Symbiotic development. At harvest ( 8 weeks after sowing), the percentage of mycorrhizal fungal colonization in maize plants was estimated by visual observation according to Phillips and Hayman (1970). The extent of mycorrhizal colonization was calculated according to the gridline intersect method (Giovannetti and Mosse 1980) in six replicates per treatment.

$J v$ and Lo. Eight weeks after sowing, Jv and Lo were measured on detached roots exuding under atmospheric pressure for $2 \mathrm{~h}$ (Aroca et al. 2007). Lo was calculated as Lo $=\mathrm{Jv} / \Delta \Psi$, where Jv is the exuded sap flow rate and $\Delta \Psi$ the osmotic potential difference between the exuded sap and the nutrient solution where the pots were immersed. These measurements were carried out $3 \mathrm{~h}$ after the onset of light.

$L h$. At harvest time, Lh was measured using a high pressure flow meter (HPFM, Dynamax, Houston), as previously described (Bárzana et al. 2012). The root conductance was measured at the transient mode. Water flow into roots and applied pressure were measured every $3 \mathrm{~s}$, the applied pressure increasing at the constant rate of 5 to $7 \mathrm{kPa} \mathrm{s}^{-1}$. Four plants per treatment (whole root system) with two measurements per plant were used in this analysis.

\section{Molecular analyses.}

Quantitative real-time reverse-transcriptase polymerase chain reaction. Total RNA was isolated from maize roots harvested 8 weeks after sowing and kept at $-80^{\circ} \mathrm{C}$ by a phenol/ chloroform extraction method followed by precipitation with $\mathrm{LiCl}$ (Kay et al. 1987). The RNA was subjected to DNase treatment and reverse-transcription using the QuantiTect reversetranscription kit (Qiagen, Hilden, Germany), following the instructions provided by manufacturer. To rule out the possibility of a genomic DNA contamination, all the cDNA sets were checked by running control polymerase chain reaction (PCR) reactions with aliquots of the same RNA that have been subjected to the DNase treatment but not to the reverse-transcription step.

The expression of the aquaporin gene family in maize was studied by real-time PCR by using iCycler system (Bio-Rad, Hercules, CA, U.S.A.), adjusting protocols to optimize the PCR reaction to each gene. The primer sets used to amplify each aquaporin gene were designed in the $3^{\prime}$ and $5^{\prime}$ untranslated regions of each gene in order to avoid unspecific amplification of the different aquaporin genes (Hachez et al. 2006). The specificity of amplicons was checked with a heat dissociation protocol (from 70 to $100^{\circ} \mathrm{C}$ ), after the final PCR cycle. The efficiency of the primer sets was evaluated with the software Bio-Rad iQ5 (version 2.1.97.1001) by analyzing the ratio threshold cycle $(\mathrm{Ct}) /$ fluorescence at four to six independent points of PCR curves (Remakers et al. 2003), giving values between 90 and $98 \%$. The sequences of primers used for the 31 maize aquaporin genes and four constitutively expressed genes are shown in Supplementary Table S1.

Standardization was carried out by calculating a normalization factor based on the three best-performing housekeeping genes. It was measured using a Visual basic application for excel (GeNorm) that automatically calculates the gene stability measure, as described by Vandesompele and associates (2002). Four reference genes were used for this calculation-poliubiquitin (gi:248338), tubulin (gi:450292), GAPDH (gi:22237), and elongation factor 1 (gi:2282583) —and the worst-scoring gene was discarded. This calculation was done for each cDNA used in the quantitative PCR quantification. Expression levels were transformed from quantification cycle values according to Die and associates (2010), using the primer efficiencies (Ramakers et al. 2003). Finally, normalized expression levels were rescaled before the statistical analysis. Real-time PCR measurements were carried out in three independent RNA samples per treatment, with the $\mathrm{Ct}$ was determined in triplicate.

Protein accumulation. Microsomes were isolate from three different root samples for each treatment, as described by Hachez and associates (2006), and kept at $-80^{\circ} \mathrm{C}$. Antibodies directed against some of the aquaporins showing a differential expression pattern between AM and non-AM treatments were designed (ZmPIP1;2, ZmPIP2;1-ZmPIP2;2, ZmPIP2;3ZmPIP2;4, ZmTIP1;1, and ZmTIP1;2). Specific antibodies against ZmPIP2;5, as one of the most expressed PIP proteins in maize roots (Hachez et al. 2006), were also used. In some cases, the antibody recognized two aquaporin isoforms that have similar amino acid sequences, as occur in pairs: ZmPIP2;1ZmPIP2;2 and ZmPIP2;3-ZmPIP2;4. The antibodies raised against ZmPIP1;2, ZmPIP2;1-ZmPIP2;2, and ZmPIP2;5 were described by Hachez and associates (2006). The sequences of peptides used to elicit the antibodies are shown in Supplementary Table S2. For aquaporin quantification, protein $(2 \mu \mathrm{g})$ was loaded in triplicate on enzyme-linked immunosorbent assay (ELISA) plates and incubated overnight in the presence of coating buffer $(0.05 \mathrm{M}$ carbonate/bicarbonate, $\mathrm{pH}$ 9.6). After that, plates were washed three times with Tris-buffered-saline containing $0.05 \%$ Tween 20 (TTBS) and were blocked during $1 \mathrm{~h}$ at room temperature with $1 \%$ bovine serum albumen (wt/vol) in TTBS, then washed again three times for $10 \mathrm{~min}$ with TTBS (Boursiac et al. 2005). The plates were then incubated at room temperature with 1:2,000 dilutions of antibodies raised against the amino-terminal peptides of goat anti-rabbit (ZmPIP1;2 and ZmPIP2;1-PIP2;2) or goat anti-chicken (ZmPIP2;3-PIP2;4, ZmTIP1;1, and ZmTIP1;2). Immunoglobulin coupled to horseradish peroxidase (Sigma, St. Louis) was used as secondary antibody at a 1:20,000 dilution. The signal was developed using a TMB substrate (Sigma) and the colorimetric reaction was produced with $2 \mathrm{M} \mathrm{H}_{2} \mathrm{SO}_{4}$. The amount of each aquaporin was quantified by measuring the intensity of signals into each well at $450 \mathrm{~nm}$ with a spectrophotometer Infinite 200 PRO series (Tecan Trading AG, Männedorf, Switzerland). Protein quantification was carried out on three microsomal samples of each treatment without significant differences among them. The equal loading of proteins in the different treatments was confirmed by staining a gel blot loaded with the same quantities used for the ELISA measurement with Coomassie brilliant blue and also by Bradford quantification.

\section{Functional characterization.}

Heterologous expression in X. laevis oocytes. cDNA encoding ZmPIP1;3-PIP1;4, ZmPIP2;2, ZmNIP1;1, ZmNIP2;1, ZmNIP2;2, ZmTIP1;1, and ZmTIP1;2 were PCR amplified and cloned into the USER-compatible Xenopus expression vector pNB1 using the USER system (uracil-specific excision 
reagent cloning technique), as described by Nour-Eldin and associates (2006). The generated constructs were sequenced.

Ready-to-use capped complementary RNAs encoding the aquaporins of interest were synthesized and injected in $X$. laevis oocytes, as described previously (Fetter et al. 2004). For water transport assays, oocyte swelling was recorded with a digital camera and the $P_{f}$ was calculated (Fetter et al. 2004).

For solute transport assays, individual oocytes were transferred from Barth's buffer (200 mOsm kg-1) to isotonic modified Barth's solutions containing glycerol $(150 \mathrm{mM})$ or boric acid $(150 \mathrm{mM})$ and the variations in volume $(\mathrm{Vo}=\mathrm{d}(\mathrm{V} / \mathrm{Vo}) / \mathrm{dt})$ quantified (Gerbeau et al. 1999; Tanaka et al. 2008). Each protein was tested in two to four different experiments with a total of 10 to 20 oocytes.

Heterologous expression in yeasts. Selected maize aquaporin genes were optimized into synthetic nucleotide DNA sequences compatible with yeast expression and cloned into pMA-T vectors by Life Technologies Corporation (Carlsbad, CA, U.S.A.).

DNA-plasmid containing optimized ZmPIP1;3-PIP1;4, ZmPIP2;2, ZmNIP1;1, ZmTIP1;1, and ZmTIP1;2 were cloned into the USER-compatible Saccharomyces cerevisiae expression vector pYeDP60u (Hamann and Møller 2007), using the USER system, as described by Nour-Eldin and associates (2006). DNA-plasmid containing nonoptimized ZmNIP2; 1 was also cloned in the same vector. The obtained constructs were sequenced. Two strains of $S$. cerevisiae were used for the complementation and toxicity growth assays: $\Delta$ mep1-3 triple mutant 31019b (Marini et al. 1997) and $\Delta$ dur3 mutant YNVW1 (Liu et al. 2003a). Competent cells of both strains were transformed with the pYeDP60u plasmid or the pYeDP60u plasmid containing the gene of interest, as described on the Ku Lueven yeast electroporation webpage.

Transformants were spotted on synthetic galactose-medium supplemented with different additives depending on the solute to be analyzed: $0.2 \%$ proline or different concentrations of $\left(\mathrm{NH}_{4}\right)_{2} \mathrm{SO}_{3}$ as a sole nitrogen source for testing $\mathrm{NH}_{3}$ transport capacity (Jahn et al. 2004), different concentrations of urea as a sole nitrogen source for testing urea transport capacity (Liu et al. 2003b), $1 \mathrm{mM}$ arginine and different concentrations of $\mathrm{GeO}_{2}$ for testing $\mathrm{Si}$ transport capacity ( $\mathrm{Gu}$ et al. 2012), different concentrations of $\mathrm{H}_{2} \mathrm{O}_{2}$ for testing its transport capacity (Bienert et al. 2007; Dynowski et al. 2008), or different concentrations of boric acid for testing B transport capacity (Bienert et al. 2011). All experiments were repeated twice and conducted at pH 5.5 (Bienert et al. 2007, 2011; Dynowski et al. 2008; Gu et al. 2012). Yeast growth was monitored after 6 to 9 days at $28^{\circ} \mathrm{C}$.

\section{ACKNOWLEDGMENTS}

This work was financed by Ministry of Science and Innovation (project AGL2008-00898), by Junta de Andalucía (project P11-CVI-07107), the Interuniversity Attraction Poles Programme-Belgian Science Policy, and the "Communauté française de Belgique-Actions de Recherches Concertées”. G. Bárzana was financed by the JAE-predoc programme (Consejo Superior de Investigaciones Científicas) and G. P. Bienert was financed by an individual fellowship from the Belgian National Fund for Scientific Research.

\section{LITERATURE CITED}

Alexandersson, E., Fraysse, L., Sjöval-Larsen, S., Gustavsson, S., Fllert, M., Karlsson, M., Johanson, U., and Kjelbom, P. 2005. Whole gene family expression and drought stress regulation of aquaporins. Plant Mol. Biol. 59:469-484

Allen, M. F. 2007. Mycorrhizal fungi: Highways for water and nutrients in arid soils. Vadose Zone J. 6:291-297.

Allen, M. F. 2009. Bidirectional water flows through the soil-fungal-plant mycorrhizal continuum. New Phytol. 182:290-293.
Aroca, R., Porcel, R., and Ruiz-Lozano J. M. 2007. How does arbuscular mycorrhizal symbiosis regulate root hydraulic properties and plasma membrane aquaporins in Phaseolus vulgaris under drought, cold or salinity stresses? New Phytol. 173:808-816.

Aroca, R., Bago, A., Sutka, M., Paz, J. A., Cano, C., Amodeo, G., and Ruiz-Lozano J. M. 2009. Expression analysis of the first arbuscular mycorrhizal fungi aquaporin described reveals concerted gene expression between salt-stressed and non-stressed mycelium. Mol. Plant-Microbe Interact. 22:1169-1178.

Augé, R. M. 2001. Water relations, drought and vesicular-arbuscular mycorrhizal symbiosis. Mycorrhiza 11:3-42.

Bansal, A., and Sankararamakrishnan, R. 2007. Homology modeling of major intrinsic proteins in rice, maize and Arabidopsis: Comparative of the selectivity filters. BMC Struct. Biol. 7:27-44.

Bárzana, G., Aroca, R., Paz, J. A., Chaumont, F., Martinez-Ballesta, M. C., Carvajal, M., and Ruiz-Lozano, J. M. 2012. Arbuscular mycorrhizal symbiosis increases relative apoplastic water flow in roots of the host plant under both well-watered and drought stress conditions. Ann. Bot. 109:1009-1017.

Benabdellah, K., Ruiz-Lozano, J. M., and Aroca, R. 2009. Hydrogen peroxide effects on root hydraulic properties and plasma membrane aquaporin regulation in Phaseolus vulgaris. Plant Mol. Biol. 70:647-661.

Bienert, G. P., Schjoerring, J. K., and Jahn, T. P. 2006. Membrane transport of hydrogen peroxide. Biochim. Biophys. Acta 1758:9941003.

Bienert, G. P., Møller, A. L. B., Kristiansen, K. A., Schulz, A., Møller, I. M., Schjoerring, J. K., and Jahn T. P. 2007. Specific aquaporins facilitate the diffusion of hydrogen peroxide across membranes. J. Biol. Chem. 282:1183-1192.

Bienert, G. P., Schüssler, M. D., and Jahn, T. P. 2008. Metalloids essential, beneficial or toxic? Major intrinsic proteins sort it out. Trends Biochem. Sci. 33:21-26.

Bienert, G. P., Bienert, M. D., Jahn, T. P., Boutry, M., and Chaumont, F. 2011. Solanaceae XIPs are plasma membrane aquaporins that facilitate the transport of many uncharged substrates. Plant J. 66:306-317.

Blee, K. A., and Anderson, A. J. 1998. Regulation of arbuscule formation by carbon in the plant. Plant J. 16:523-530.

Blevins, D. G., and Lukaszewski, K. M. 1998. Boron in plant structure and function. Annu. Rev. Plant Physiol. Plant Mol. Biol. 49:481-500.

Boomsma, C. R., and Wyn, T. J. 2008. Maize drought tolerance: Potential improvements through arbuscular mycorrhizal symbiosis? Field Crop Res. 108:14-31.

Boursiac, Y., Boudet, J., Postaire, O., Luu, D.-T., Tournaire-Roux, C., and Maurel, C. 2008. Stimulus-induced downregulation of root water transport involves reactive oxygen species-activated cell signaling and plasma membrane intrinsic protein internalizatization. Plant J. 56:207218.

Boursiac, Y., Chen, S., Luu, D.-T., Sorieul, M., Dries, N., and Maurel, C. 2005. Early effects of salinity on water transport in Arabidopsis roots. Molecular and cellular features of aquaporin expression. Plant Physiol. 139:790-805

Cabello-Hurtado, F., and Ramos, J. 2004 Isolation and functional analysis of the glycerol permease activity of two new nodulin-like intrinsic proteins from salt stressed roots of the halophyte Atriplex nummularia. Plant Sci. 166:633-640.

Campos, H., Cooper, M., Habben, J. E., Edmeades, G. O., and Schussler, J. R. 2004. Improving drought tolerance in maize: A view from industry. Field Crop Res. 90:19-34.

Chaumont, F., Barrieu, F., Jung, R., and Chrispeels, M. J. 2000. Plasma membrane intrinsic proteins from maize cluster in two sequence subgroups with differential aquaporin activity. Plant Physiol. 122:10251034.

Chaumont, F., Barrieu, F., Wojcik, E., Chrispeels, M. J., and Jung, R. 2001. Aquaporins constitute a large and highly divergent protein family in maize. Plant Physiol. 125:1206-1215.

Chaves, M. M., and Oliveira, M. M. 2004. Mechanisms underlying plant resilience to water deficits: Prospects for water-saving agriculture. J. Exp. Bot. 55:2365-2384

Die, J. V., Román, B., Nadal, S., and González-Verdejo, C. I. 2010. Evaluation of candidate reference genes for expression studies in Pisum sativum under different experimental conditions. Planta 232:145-153.

Dietz, S., Von Bülow, J., Beitz, E., and Nehls, U. 2011. The aquaporin gene family of the ectomycorrhizal fungus Laccaria bicolor: Lessons for symbiotic functions. New Phytol. 190:927-940.

Dynowski, M., Schaaf, G., Loque, D., Moran, O., and Ludewig, U. 2008 Plant plasma membrane water channels conduct the signaling molecule $\mathrm{H}_{2} \mathrm{O}_{2}$. Biochem. J. 414:53-61.

Farooq, M., Aziz, T., Wahid, A., Lee, D.-J., and Siddique, K. H. M. 2009. Chilling tolerance in maize: Agronomic and physiological approaches. Crop Pasture Sci. 60:501-516. 
Fauteux, F., Rémus-Borel, W., Menzies, J. G., and Bélanger, R. R. 2005. Silicon and plant disease resistance against pathogenic fungi. FEMS (Fed. Eur. Microbiol. Soc.) Microbiol. Lett. 249:1-6.

Fetter, K., Van Wilder, V., Moshelion, M., and Chaumont, F. 2004. Interactions between plasma membrane aquaporins modulate their water channel activity. Plant Cell 16:215-228.

Forrest, K. L., and Bhave, M. 2007. Major intrinsic proteins (MIPs) in plants: A complex gene family with impact on plant phenotype. Funct. Integr. Genom. 7:263-289.

Galmés, J., Medrano, H., and Flexas, J. 2007. Photosynthetic limitations in response to water stress and recovery in Mediterranean plants with different growth forms. New Phytol. 175:81-93.

Gerbeau, P., Güçlü, J., Ripoche, P., and Maurel, C. 1999. Aquaporin NtTIPa can account for the high permeability of tobacco cell vacuolar membrane to small neutral solutes. Plant J. 18:577-587.

Giovannetti, M., and Mosse, B. 1980. An evaluation of techniques for measuring vesicular-arbuscular infection in roots. New Phytol. 84:489500 .

Govindarajulu, M., Pfeffer, P. E., Jin, H., Abubaker, J., Douds, D. D., Allen, J. W., Bücking, H., Lammers, P. J., and Shachar-Hill, Y. 2005. Nitrogen transfer in the arbuscular mycorrhizal symbiosis. Nature 435:819-823.

Gu, R., Chen, X., Zhou, Y., and Yuan, L. 2012. Isolation and characterization of three maize aquaporin genes ZmNIP2;1, ZmNIP2;4 and ZmTIP4;4 involved in urea transport. BMB Rep. 45:96-101.

Gustavsson, S., Lebrun, A.-S., Nordén, K., Chaumont, F., and Johanson, U. 2005. A novel plant major intrinsic protein in Physcomitrella patens most similar to bacterial glycerol channels. Plant Physiol. 139:287-295.

Hachez, C., Moshelion, M., Zelazny, E., Cavez, D., and Chaumont, F. 2006. Localization and quantification of plasma membrane aquaporin expression in maize primary root: A clue to understanding their role as cellular plumbers. Plant Mol. Biol. 62:305-323.

Hamann, T., and Møller, B. L. 2007. Improved cloning and expression of cytochrome P450s and cytochrome P450 reductase in yeast. Protein Express. Purif. 56:121-127.

Hunter, P. R., Craddock, C. P., Di Benedetto, S., Roberts, L. M., and Frigerio, L. 2007. Fluorescent reporter proteins for the tonoplast and the vacuolar lumen identify a single vacuolar compartment in Arabidopsis cells. Plant Physiol. 145:1371-1382.

Jahn, T. P., Møller, A. L. B., Zeuthenb, T., Holm, L. M., Klærke, D. A., Mohsinc, B., Kühlbrandt, W., and Schjoerring, J. K. 2004. Aquaporin homologues in plants and mammals transport ammonia. FEBS (Fed. Eur. Biochem. Soc.) Lett. 574:31-36.

Javot, H., and Maurel, C. 2002. The role of aquaporins in root water uptake. Ann. Bot. 90:301-313.

Katsuhara, M., Hanba, Y. T., Shiratake, K., and Maeshima, M. 2008. Expanding roles of plant aquaporins in plasma membranes and cell organelles. Funct. Plant Biol. 35:1-14.

Kay, R., Chau, A., and Daly, M. 1987. Duplication of CaMV 35S promoter sequences creates a strong enhancer for plants genes. Science 236:1299-1302.

Khalvati, M. A., Hu, Y., Mozafar, A., and Schmidhalter, U. 2005. Quantification of water uptake by arbuscular mycorrhizal hyphae and its significance for leaf growth, water relations, and gas exchange of barley subjected to drought stress. Plant Biol. 7:706-712.

Koide, R. T., and Mosse, B. 2004. A history of research on arbuscular mycorrhiza. Mycorrhiza 14:145-163.

Krajinski, F., Biela, A., Schubert, D., Gianinazzi-Pearson, V., Kaldenhoff, R., and Franken, P. 2000. Arbuscular mycorrhiza development regulates the mRNA abundance of Mtaqp1 encoding a mercury-insensitive aquaporin of Medicago truncatula. Planta 211:85-90.

Lehto, T., and Zwiazek, J. J. 2011. Ectomycorrhizas and water relations of trees: A review. Mycorrhiza 21:71-90.

Lehto, T., Ruuhola, T., and Dell, B. 2010. Boron in forest trees and forest ecosystems. For. Ecol. Manage. 260:2053-2069.

Li, T., Hu, Y.-J., Hao, Z.-P., Li, H., Wang, Y.-S., and Chen, B.-D. 2013. First cloning and characterization of two functional aquaporin genes from an arbuscular mycorrhizal fungus Glomus intraradices. New Phytol. 197:617-630.

Liu, L. H., Ludewig, U., Frommer, W. B., and Von Wirén, N. 2003a. AtDUR3 encodes a new type of high-affinity urea/ $\mathrm{H}^{+}$symporter in Arabidopsis. Plant Cell 15:790-800.

Liu, L. H., Ludewig, U., Gassert, B., Frommer, W. B., and Von Wirén, N. 2003b. Urea transport by nitrogen-regulated tonoplast intrinsic proteins in Arabidopsis. Plant Physiol. 133:1220-1228.

Loque, D., Ludewig, U., Yuan, L., and Von Wirén, N. 2005. Tonoplast intrinsic proteins AtTIP2;1 and AtTIP2;3 facilitate $\mathrm{NH}_{3}$ transport into the vacuole. Plant Physiol. 137:671-680.

Lovisolo, C., Secchi, F., Nardini, A., Salleo, S., Buffa, R., and Schubert, A. 2007. Expression of PIP1 and PIP2 aquaporins is enhanced in olive dwarf genotypes and is related to root and leaf hydraulic conductance.
Physiol. Plant. 130:543-551.

Luu, D.-T., and Maurel, C. 2005. Aquaporins in a challenging environment: Molecular gears for adjusting plant water status. Plant Cell Environ. 28:85-96.

Ma, J. F., and Yamaji, N. 2006. Silicon uptake and accumulation in higher plants. Trends Plant Sci. 11:392-397.

Ma, J. F., and Yamaji, N. 2008. Functions and transport of silicon in plants. Cell Mol. Life Sci. 65:3049-3057.

Marini, A. N., Soussi-Boudekou, S., Vissers, S., and Andre, B. 1997. A family of ammonium transporters in Sacharomyces cerevisiae. Mol. Cell Biol. 17:4282-4293.

Maurel, C. 2007. Plant aquaporins: Novel functions and regulation properties. FEBS (Fed. Eur. Biochem. Soc.) Lett. 581:2227-2236.

Maurel, C., and Plassard, C. 2011. Aquaporins. For more than water at the plant fungus interface. New Phytol. 190:815-817.

Maurel, C., Verdoucq, L., Luu, D. T., and Santoni, V. 2008. Plant aquaporins: Membrane channels with multiple integrated functions. Annu. Rev. Plant Biol. 59:595-624.

Miller, G., Suzuki, N., Ciftci-Yilmaz, S., and Mittler, R. 2010. Reactive oxygen species homeostasis and signaling during drought and salinity stress. Plant Cell Environ.33:453-467.

Mitani, N., Yamaji, N., and Ma, J. F. 2008. Characterization of substrate specificity of a rice silicon transporter, Lsi1. Eur. J. Physiol. 456:679686.

Mitani, N., Chiba, Y., Yamaji, N., and Ma, J. F. 2009. Identification and characterization of maize and barley Lsi2-like silicon efflux transporters reveals a distinct silicon uptake system from that in rice. Plant Cell 21:2133-2142.

Miwa, K., Kamiya, T., and Fujiwara, T. 2009. Homeostasis of the structurally important micronutrients, B and Si. Curr. Opin. Plant Biol. 12:307311.

Muhsin, T. M., and Zwiazek, J. J. 2002. Ectomycorhizas increase apoplastic water transport and root hydraulic conductivity in Ulmus americana seedlings. New Phytol. 153:153-158.

Nour-Eldin, H. H., Hansen, B. G., Nørholm, M. H. H., Jensen, J. K., and Halkier, B. A. 2006. Advancing uracil-excision based cloning towards an ideal technique for cloning PCR fragments. Nucleic Acids Res. 34:18-26.

Ouziad, F., Wilde, P., Schmelzer, E., Hildebrandt, U., and Bothe, H. 2006. Analysis of expression of aquaporins and $\mathrm{Na}^{+} / \mathrm{H}^{+}$transporters in tomato colonized by arbuscular mycorrhizal fungi and affected by salt stress. Environ. Exp. Bot. 57:177-186.

Park, W., Scheffler, B. E., Bauer, P. J., and Campbell, B. T. 2010. Identification of the family of aquaporin genes and their expression in upland cotton (Gossypium hirsutum L.). BMC Plant Biol. 10:142-159.

Pérez-Tienda, J., Testillano, P. S., Balestrini, R., Valentina Fiorilli, V., Azcón-Aguilar, C., and Ferrol, N. 2011. GintAMT2, a new member of the ammonium transporter family in the arbuscular mycorrhizal fungus Glomus intraradices. Fungal Genet. Biol. 48:1044-1055.

Phillips, J. M., and Hayman, D. S. 1970. Improved procedure of clearing roots and staining parasitic and vesicular-arbuscular mycorrhizal fungi for rapid assessment of infection. Trans. Br. Mycol. Soc. 55:159-161.

Porcel, R., and Ruiz-Lozano, J. M. 2004. Arbuscular mycorrhizal influence on leaf water potential, solute accumulation and oxidative stress in soybean plants subjected to drought stress. J. Exp. Bot. 55:1743-1750.

Porcel, R., Aroca, R., Azcón, R., and Ruiz-Lozano J. M. 2006. PIP aquaporin gene expression in arbuscular mycorrhizal Glycine max and Lactuca sativa plants in relation to drought stress tolerance. Plant Mol. Biol. 60:389-404.

Quan, L.-J., Zhang, B., Shi, W.-W., and Li, H.-Y. 2008. Hydrogen peroxide in plants: A versatile molecule of the reactive oxygen species network. J. Integr. Plant Biol. 50:2-18.

Ramakers, C., Ruijter, J. M., Deprez, R. H. L., and Moorman, A. F. M. 2003. Assumption-free analysis of quantitative real-time polymerase chain reaction (PCR) data. Neurosci. Lett. 339:62-66.

Ruiz-Lozano, J. M. 2003. Arbuscular mycorrhizal symbiosis and alleviation of osmotic stress. New perspectives for molecular studies. Mycorrhiza 13:309-317

Ruiz-Lozano, J. M., and Aroca, R. 2010. Modulation of aquaporin genes by the arbuscular mycorrhizal symbiosis in relation to osmotic stress tolerance. Pages 359-374. In: Symbioses and Stress: Joint Ventures in Biology, Cellular Origin, Life in Extreme Habitats and Astrobiology. J. Seckbach and M. Grube, eds. Springer Science+Business Media, Dordrecht, The Netherlands.

Ruiz-Lozano, J. M., Porcel, R, and Aroca, R. 2006. Does the enhanced tolerance of arbuscular mycorrhizal plants to water deficit involve modulation of drought-induced plant genes? New Phytol. 171:693-698.

Ruiz-Lozano, J. M., Alguacil, M. M., Bárzana, G., Vernieri, P., and Aroca, R. 2009. Exogenous ABA accentuates the differences in root hydraulic properties between mycorrhizal and non mycorrhizal maize plants 
through regulation of PIP aquaporins. Plant Mol. Biol. 70:565-579.

Ruiz-Lozano, J. M., Porcel, R., Azcón, R., and Aroca, R. 2012. Regulation by arbuscular mycorrhizae of the integrated physiological response to salinity in plants: New challenges in physiological and molecular studies. J. Exp. Bot. 63:4033-4044.

Smith, S. E., Facelli, E., Pope, S., and Smith, F. A. 2010. Plant performance in stressful environments: Interpreting new and established knowledge of the roles of arbuscular mycorrhizas. Plant Soil 326:3-20.

Sui, H., Han, B. G., Lee, J. K., Walian, P., and Jap, B. K. 2001. Structural basis of water-specific transport through AQP1 water channel. Nature 414:872-878.

Tallberg, P., Koski-Vahala, J., and Hartikainen, H. 2002. Germanium-68 as a tracer for silicon fluxes in freshwater sediment. Water Res. 36:956962.

Tanaka, M., Wallace, I. S., Takano, J., Roberts, D. M., and Fujiwara, T. 2008. NIP6;1 is a boric acid channel for preferential transport of boron to growing shoot tissues in Arabidopsis. Plant Cell 20:2860-2875.

Tian, C., Kasiborski, B., Koul, R., Lammers, P. J., Bucking, H., and ShacharHill, Y. 2010. Regulation of the nitrogen transfer pathway in the arbuscular mycorrhizal symbiosis: Gene characterization and the coordination of expression with nitrogen flux. Plant Physiol. 153:1175-1187.

Uehlein, N., Lovisolo, C., Siefritz, F., and Kaldenhoff, R. 2003. The tobacco aquaporin NtAQP1 is a membrane $\mathrm{CO}_{2}$ pore with physiological functions. Nature 425:734-737.

Uehlein, N., Fileschi, K., Eckert, M., Bienert, G. P., Bertl, A., and Kaldenhoff, R. 2007. Arbuscular mycorrhizal symbiosis and plant aquaporin expression. Phytochemistry 68:122-129.

Vandesompele, J., De Preter, K., Pattyn, F., Poppe, B., Van Roy, N., De Paepe, A., and Speleman, F. 2002. Accurate normalization of real-time quantitative RT-PCR data by geometric averaging of multiple internal control genes. Genome Biol. 3:research0034.

Varma, A. 2008. Mycorrhiza. State of the art, Genetics and Molecular Biology, Eco-Funcion, Biotecnology, Eco-Physiology, Structure and Systematics, third ed. Springer-Verlag, Berlin, Heidelberg.
Vera-Estrella, R., Barkla, B. J., Bohnert, H. J., and Pantoja, O. 2004. Novel regulation of aquaporins during osmotic stress. Plant Physiol. 135:23182329.

Wallace, I. S., and Roberts, D. M. 2004. Homology modeling of representative subfamilies of Arabidopsis major intrinsic proteins. Classification based on the aromatic/arginine selectivity filter. Plant Physiol. 135:1059-1068.

Wallace, I. S., Wills, D. M., Guenther, J. F., and Roberts, D. M. 2002. Functional selectivity for glycerol of the nodulin 26 subfamily of plant membrane intrinsic proteins. FEBS (Fed. Eur. Biochem. Soc.) Lett. 523:109-112.

Wang, W.-H., Köhler, B., Cao, F.-Q., and Liu, L. H. 2008. Molecular and physiological aspects of urea transport in higher plants. Plant Sci. 175:467-477.

Wei, Y., Shen, W., Dauk, M., Wang, F., Selvaraj, G., and Zou, J. 2004. Targeted gene disruption of glycerol-3-phosphate dehydrogenase in Colletotrichum gloeosporioides reveals evidence that glycerol is a significant transferred nutrient from host plant to fungal pathogen. J. Biol. Chem. 279:429-435

Weig, A. R., and Jakob, C. 2000. Functional identification of the glycerol permease activity of Arabidopsis thaliana NLM1 and NLM2 proteins by heterologous expression in Saccharomyces cerevisiae. FEBS (Fed. Eur. Biochem. Soc.) Lett. 481:293-298.

Ye, Q., and Steudle, E. 2006. Oxidative gating of water channels (aquaporins) in corn roots. Plant Cell Environ. 29:459-470.

Zardoya, R., Ding, X., Kitagawa, Y., and Chrispeels, M. J. 2002. Origin of plant glycerol transporters by horizontal gene transfer and functional recruitment. Proc. Natl. Acad. Sci. U.S.A. 99:14893-14896.

\section{AUTHOR-RECOMMENDED INTERNET RESOURCE}

$\mathrm{Ku}$ Lueven yeast electroporation webpage: www.agr.kuleuven.ac.be/dp/logt/protocol/yeastelectroporation.htm 